\title{
The Planar Wide-Frequency Vibration Characteristics of Heavy-Load Radial Tires
}

\author{
Hongjie Cheng, Lei Gao, Zhihao Liu (iD, Qinhe Gao ${ }^{D}$, and Xiuyu Liu
}

Xi'an Research Institution of High Technology, Xi'an 710025, China

Correspondence should be addressed to Zhihao Liu; liuzh_1989@126.com

Received 22 May 2020; Revised 16 August 2020; Accepted 24 August 2020; Published 23 April 2021

Academic Editor: Mijia Yang

Copyright ( $\odot 2021$ Hongjie Cheng et al. This is an open access article distributed under the Creative Commons Attribution License, which permits unrestricted use, distribution, and reproduction in any medium, provided the original work is properly cited.

\begin{abstract}
This paper investigates the planar wide-frequency vibration characteristics of heavy-load radial tires with a large aspect ratio. A proposed tire model with a piecewise flexible beam on an elastic foundation is investigated and validated using experimental modal analysis and theoretical modeling method. The reproducibility of frequency response functions below $400 \mathrm{~Hz}$ is discussed. The experimental modal analysis particularly assesses the coupling of features across the circumferential and cross-sectional directions of heavy-load radial tire carcass. Piecewise circumferential modal characteristics were investigated experimentally, leading to the suggestion of a piecewise flexible beam on an elastic tire foundation. Using a genetic algorithm (GA), the structural parameters $E I, \rho A$, and $k_{r}$ and damping coefficients $\eta$ and $c_{r}$ for the proposed tire model are identified, and the piecewise transfer functions and the planar transfer functions for a heavy-load radial tire are compared with planar hammer test. Experimental and theoretical results show the following: (1) the sectional vibration characteristics for a heavy-load radial tire with a large aspect ratio result from the cross-sectional vibration of the tire carcass; (2) the piecewise transfer function is mainly influenced by the circumferential vibration of the flexible carcass, and this is consistent with a model where a flexible beam is placed on an elastic tire foundation; (3) the analytical transfer functions calculated for the proposed tire model, drawing on the identified structural parameters and damping coefficients, agree well with the experimental results.
\end{abstract}

\section{Introduction}

As the only link interacting with the road, heavy-load radial tires are required to perform multiple functions, such as supporting the vehicle weight, cushioning against the road irregularities, and providing the desired braking/traction/ lateral force for vehicle control systems. In recent years, as operating speeds have increased [1], driving characteristics, such as safety [2], maneuverability [3], and ride comfort [4], have all become more important in the automotive community. At the same time, the kinematic modeling of tire components [5] and force prediction relating to road-tire interaction [6] have proved to be of abiding interest. However, multibody simulation of these features remains a challenge.

Simplified tire models, including both empirical/semiempirical ones [7] and physical ones [8], characterize force/ moment characteristics using constructed functions and analytic boundary conditions with many physical assumptions. Whilst they are computationally efficient, these kinds of tire models do not necessarily produce reliable results and usually disregard the structural flexibility of tires.

At the other extreme, as computational numerical simulation has developed, finite element analysis- (FEA) based tire models, such as a nonlinear finite element tire model [9], an absolute nodal coordinate formulation (ANCF) finite element tire model [10], and a spectral element formulation tire model [11], have all been introduced to assist with the detailed analysis of the structural and material properties of tires.

This has also helped with detailed research regarding coupled vibration, contact stress, and wear [6] between the tire and the road. Detailed tire models are now widely used for the structural design and geometric optimization of tires. However, FEA involves significant amounts of computation and preprocessing, making the detailed simulation of the 
impact of different tire models on vehicle dynamics difficult to realize.

In order to find a balance between the computational demands of detailed tire models and the lower accuracy of simplified tire models, various mechanical structure-based tire models have been proposed, with much research being dedicated to improving simulation accuracy and numerical efficiency during theoretical modeling. The basis of these mechanical structure-based tire models is the placing of a flexible carcass upon an elastic tire foundation. "Elastic foundation" refers to the tire sidewall's inflation pressure. Flexible tire carcass models [12] can take both material nonlinearity and geometric nonlinearity into consideration [13]. Carcass deformation [14] and planar vibration [15] can then be derived using either analytic or numerical solutions, and the model can be extended to cover vehicle system dynamics. Significant research has been dedicated to the development of different flexible carcass models over the past decade. Inflation pressure can be modeled as the axle pretensile force using a tensile string tire model [16], enabling bending deformation of the tire carcass to then be ignored. The Euler beam tire model [17], however, takes both inflation pressure and bending deformation into consideration and suggests that inflation pressure and bending stiffness of the flexible carcass are the main factors causing planar vibration within the medial frequency band. The shearing action of the carcass is added to enrich the Timoshenko beam tire model [18], which is of vital importance for high-frequency vibration above $1 \mathrm{KHz}$. The tensile string tire model, Euler beam tire model, and Timoshenko beam tire model all belong to a two-dimensional class of ring tire models [19].

The flexible deformation of tire carcass mainly focuses on existing tire models, with the sidewall being modeled using a spring with a constant stiffness. This is effective for analyzing the planar vibration of tires for cars, buses, and trucks with a small aspect ratio. Tires with a small aspect ratio have a high cross-sectional stiffness, so cross-sectional vibration can be ignored. However, in the case of heavy-load radial tires, a soft cross-sectional stiffness makes the crosssectional vibration of the tire carcass much more pronounced and should be discussed further. Research using experimental modal analysis and kinematic modeling of heavy-load radial tires with a larger aspect ratio is hard to find. When it comes to the structural features of heavy-load radial tires with a larger aspect ratio, the coupled characteristics between cross-sectional vibration and circumferential vibration [20] need to be considered. This is quite distinct from analysis focused on car, bus, and truck tires with a small aspect ratio.

In this paper, we use experimental modal analysis and employ a theoretical model to investigate the planar vibration characteristics of heavy-load radial tires with a large aspect ratio. First of all, taking the cross-sectional vibration of the tire carcass into consideration, we undertake a planar experimental modal analysis. After this, we model the placement of a piecewise flexible beam on an elastic tire foundation, exploring the damping characteristics of the proposed model, including the structural damping of the flexible carcass and the proportional damping of the sidewall. In addition, previously unknown parameters of the piecewise tire model are identified, based on experimental and analytical modal resonance frequencies and planar transfer functions. Finally, planar vibration characteristics, including the piecewise transfer function and the planar transfer function, are predicted and compared to the experimental results.

\section{Planar Modal Analysis Using Cross- Sectional Features}

The heavy-load radial tire, GL07A ADVANCE, is a typical heavy-load tire, and its average axial load is usually in the range of $10-13 \mathrm{t}$. A large aspect ratio (the value of the sidewall radii divided by the tread width) is needed to ensure it can operate off-road. In this case, the aspect ratio is close to 1.

In the following sections, we present how the planar transfer function for this tire was researched experimentally using a hammer test and experimental modal analysis of the tire carcass, taking the cross-sectional vibration of the tire carcass into consideration.

2.1. Hammer Test of the Middle Line. An experimental hammer test was implemented, and the principal features are shown in Figure 1(a). Considering the uncoupled characteristic of the planar and out-plane vibration, vibration excitation was realized in the radial direction using a $\mathrm{B} \& \mathrm{~K}$ hammer sensor, with the input force of the hammer sensor being measured by a force transducer, as illustrated in Figure 1(b). In this paper, the planar vibration of the heavyload tire is researched. A PCB accelerometer sensor was used to measure the resulting acceleration of the tire carcass. This was attached to the tire with a thin layer of mounting wax. By measuring the excitation force with the hammer sensor and the vibration response with the acceleration sensor, the input excitation force and output response acceleration were gathered using high-speed data acquisition equipment, namely, a DEWE-43. Point A in Figure 1(b) is defined as the excitation and response point, with the experimental transfer function being calculated using spectrum analysis by combining the input excitation force and the output response acceleration.

In order to further improve the data quality for the measured transfer function, two additional methods were implemented: first of all, linear averaging, which was used to calculate the transfer function for every excitation point by averaging 10 repetitions of transfer function measurement; and, on top of this, so as to assess the measurement process for every excitation, the coherence function was checked to evaluate the quality of the measured transfer function.

Figure 2 shows the frequency transfer function for a heavy-load radial tire with a large aspect ratio to illustrate the vibration characteristics. Figure 2(a) illustrates the threedimensional planar transfer function between the excitation at point $\mathrm{A}$ and the response. Figure 2(b) presents the 


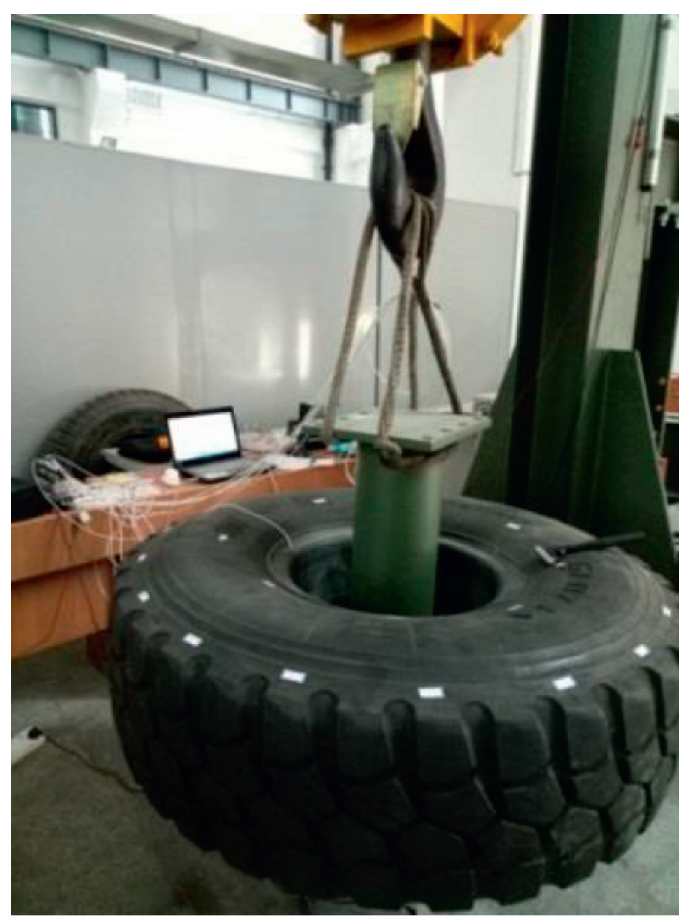

(a)

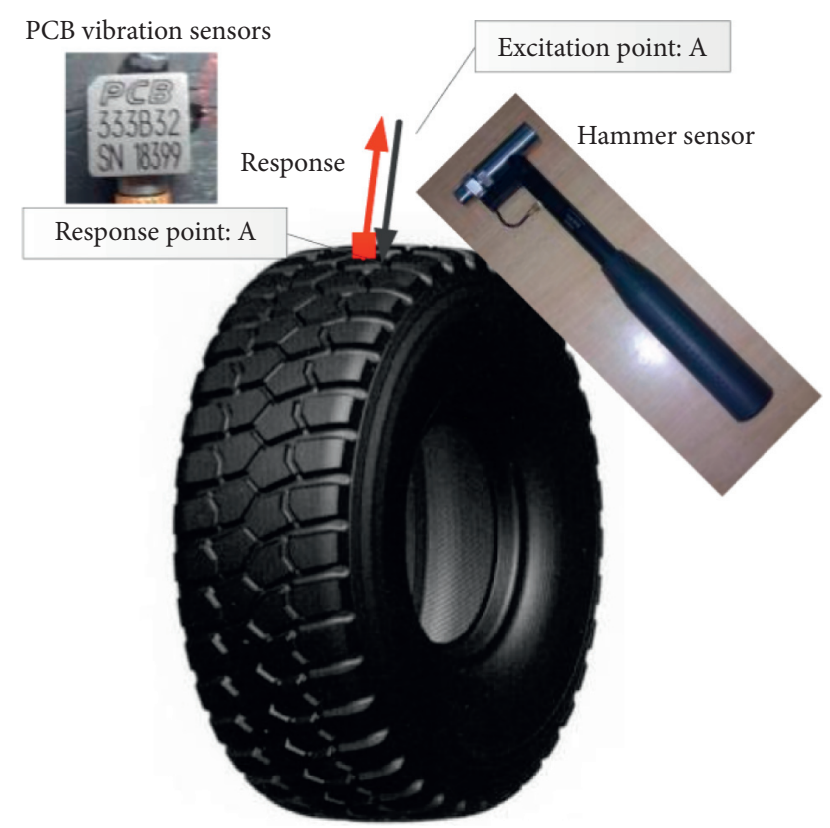

(b)

FIGURE 1: Hammer test of the cross-sectional features: (a) implementation of the experimental hammer test; (b) schematic of the hammer test.

coherence value calculated from the input force and output acceleration response as a means of checking the planar transfer function. Figures $2(\mathrm{c})-2(\mathrm{e})$ show, respectively, the first, second, and third piecewise transfer function for the planar vibration within different frequency bands. On the basis of Figure 2, we can make the following observations:

(1) The three-directional transfer function and coherence values for the excitation force and the response acceleration compared in Figure 2(a) show that the coherence value of the radial excitation force and the radial acceleration response have a difference of 1 for frequencies below $13 \mathrm{~Hz}$. There is a similar difference of 1 between the coherence value for the radial excitation force and the tangential acceleration response for frequencies below $50 \mathrm{~Hz}$. Outside of this, the coherence value of the radial excitation force and the lateral acceleration response fluctuate around the frequency of $75 \mathrm{~Hz}$

(2) The amplitude of the radial transfer function is larger than the tangential and lateral transfer functions, implying that planar radial deformation of the flexible carcass is the main source of vibration for heavy-load radial tires with a large aspect ratio.

In addition to this, the sectional planar transfer function within $400 \mathrm{~Hz}$ can be divided into three parts: $\quad 0-160 \mathrm{~Hz}$ (Figure 2(c)), $160-280 \mathrm{~Hz}$ (Figure 2(d)), and $280-360 \mathrm{~Hz}$ (Figure 2(e)), which is seen from Figure 2(a). The sectional transfer function results for the cross-sectional vibration of the carcass indicate that cross-sectional vibration generally needs to be considered and be further researched when undertaking vibration analysis of heavy-load radial tires with a large aspect ratio.

(3) The structure-cavity coupled vibration peaks at $120 \mathrm{~Hz}$ (the first-order structure-cavity resonance frequency), $240 \mathrm{~Hz}$ (the second-order structurecavity resonance frequency), and $360 \mathrm{~Hz}$ (the thirdorder structure-cavity resonance frequency), as shown in Figures 2(c)-2(e). For comparison, the first-order resonance frequency for tires with a small aspect ratio, including bus and truck tires, is typically within $230-250 \mathrm{~Hz}$ [20].

\subsection{Planar Modal Analysis Experiment Using Cross-Sectional} Features. The frequency-domain behavior of heavy-load radial tires can be used to characterize tire vibration, as shown in Figure 2. Tire modal characteristics become even more important in vehicles with $\mathrm{NVH}$ refinements. The modal characteristics of general car tires with a small aspect ratio are consistent with beam/ring vibration characteristics, and a circumferential vibration modal method will suffice in such a case. However, for tires with a large aspect ratio, the coupled characteristics between the circumferential and cross-sectional vibration are accentuated and therefore also need to be taken properly into consideration.

2.2.1. System Implementation. Considering the structural features of heavy-load radial tires, we implemented a planar modal analysis method for both the circumferential and 


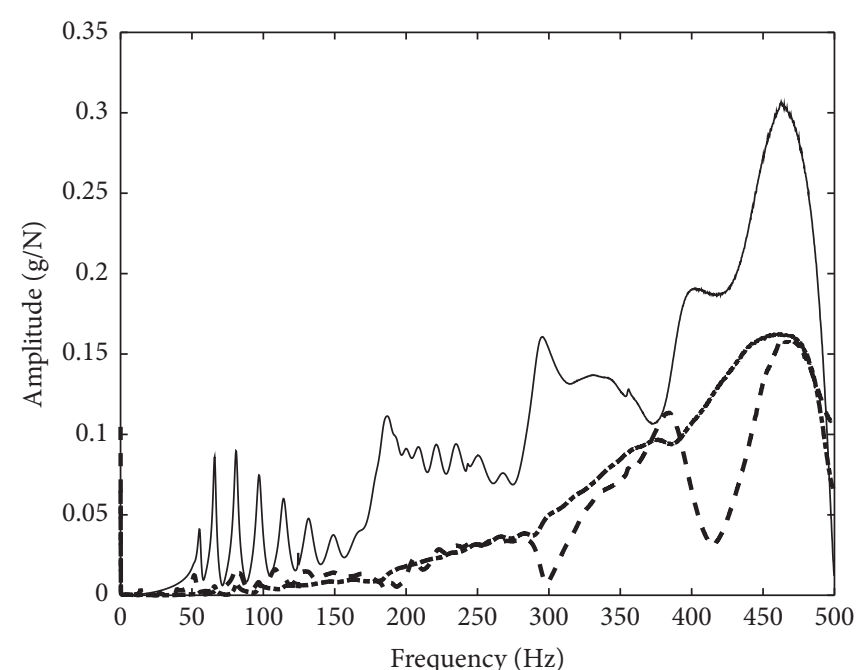

Radial transfer function

---- Tangential transfer function

- - - Lateral transfer function

(a)

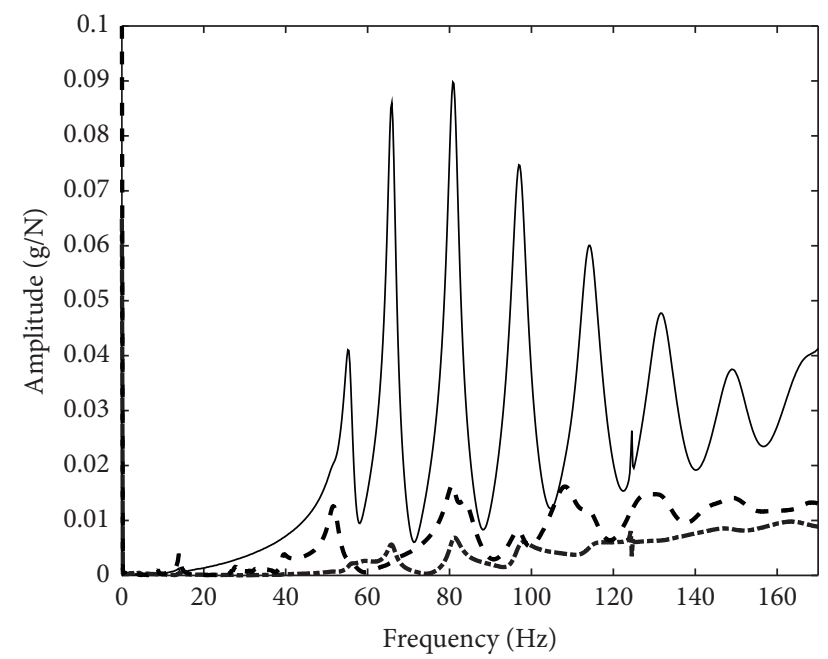

Radial transfer function

---- Tangential transfer function

- - - Lateral transfer function

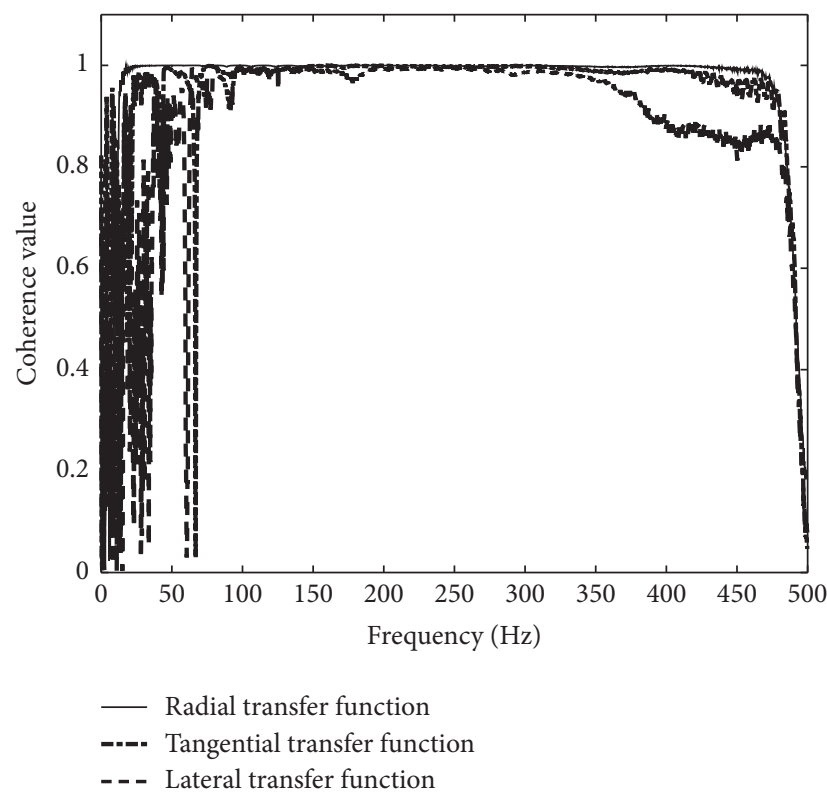

(b)

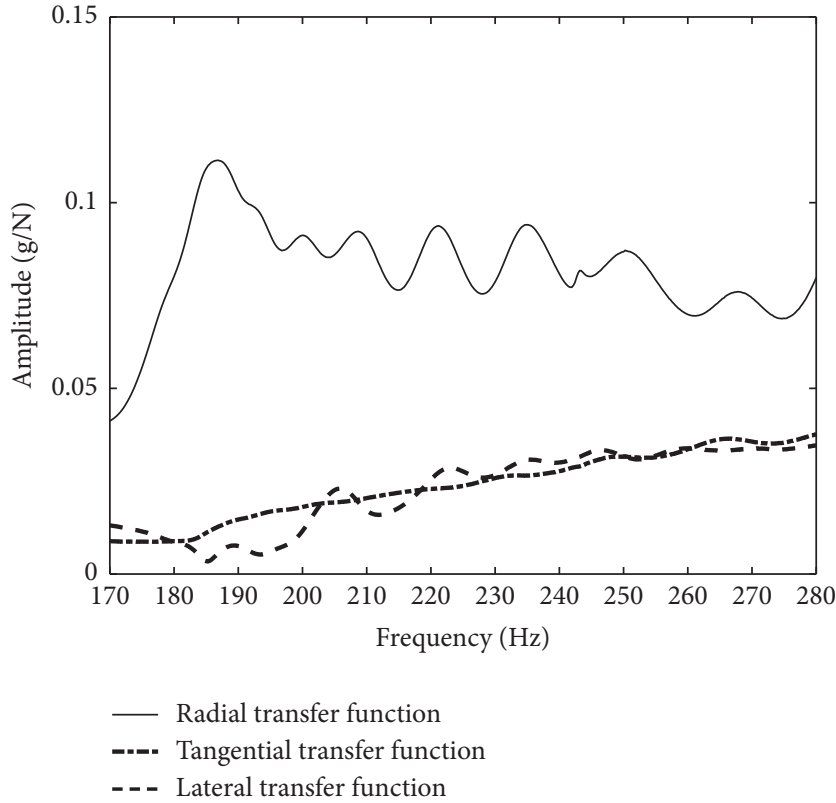

(c)

(d)

Figure 2: Continued. 


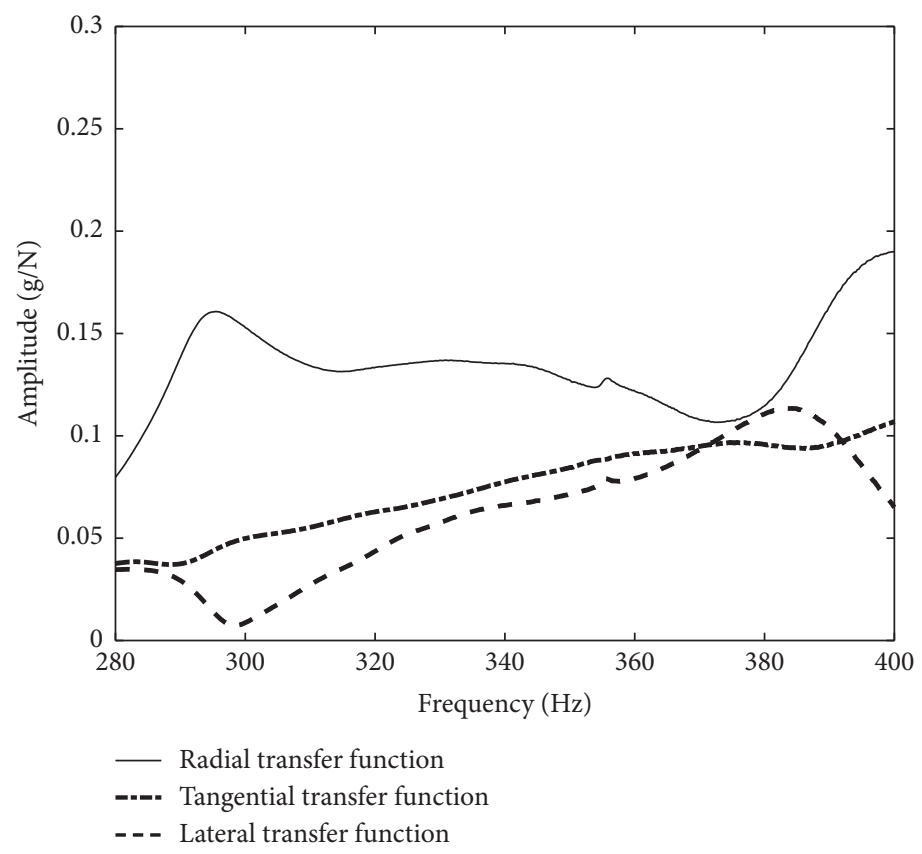

(e)

FIGURE 2: Planar acceleration transfer functions for a heavy-load radial tire with normal inflation pressure: (a) three-dimensional carcass transfer function; (b) coherence values for the three-dimensional carcass; (c) first piecewise transfer function $(0-160 \mathrm{~Hz}$ ); (d) second piecewise transfer function $(160-280 \mathrm{~Hz})$; (e) third piecewise transfer function $(280-360 \mathrm{~Hz})$.

cross-sectional vibration of a tire carcass. Figure 3(a) shows an outline of the method, with the flexible carcass being divided into $17^{*} 11$ (which is decided by the number of the tire pattern (34)) parts with 17 sections along the circumferential direction and 11 sections in the cross-sectional direction. Figure 3(b) presents the hardware used within the experimental modal system, including a tire support device, a force hammer, a charge amplifier, data acquisition equipment, and a computer. By means of moving the hammer, we were able to use the experimental modal method to investigate the circumferential and crosssectional characteristics of the flexible carcass.

The quantity of data used for the transfer function measurement directly determines the quality of the modal parameters. A linear averaging method also had to be used to obtain an average result, thereby eliminating the influence of any random errors or human interference. Once again, a coherence function was used to evaluate the quality of the transfer function measurement.

The modal analysis experiment was implemented as follows: first of all, the flexible vibration of the tire carcass was focused on, and the fixed rim was defined, with a ground to isolate the influence of any rim freedom on the tire vibration. Secondly, a moving hammer method was adopted, with the modal test incorporating one response point and $7^{*} 11$ excitation points, as shown in Figure $3(\mathrm{a})$. Outside of this, the input force was done in the radial direction and was measured using a B\&K force hammer transducer, and the signal was conditioned by a charge amplifier. The excitation force and response acceleration were collected by a DE-43 data collector, and the desired transfer function was computed using spectral analysis. The experimental transfer functions were then summed up and treated as the object transfer function. Lastly, the least squares complex exponential method (LSCE) was used to estimate the frequency, damping, participation factor, and modal shape for heavyload radial tires with a large aspect ratio by fitting the object transfer function.

2.2.2. Modal Analysis Experiment Incorporating the Circumferential and Cross-Sectional Features of a Tire Carcass. Taking into account the coupling characteristics of the circumferential and cross-sectional vibration of the tire carcass, the experimental modal parameters for a heavy-load radial tire with a large aspect ratio, including the modal resonance frequency, modal damping, and modal shape, are shown in Figures 4-7.

Figure 4 shows the eight calculated modal resonance frequencies for the first piecewise transfer function $(0-160 \mathrm{~Hz})$. The modal shapes are shown in Figure 5.

Circumferential vibration is the main kind of vibration across the shifting and two-sectional, threesectional, four-sectional, five-sectional, six-sectional, seven-sectional, and eight-sectional modal shapes, as can be seen in Figures 5(a)-5(h). Shifting vibration within the carcass's cross-section, however, does also exist.

From the experiment examining the modal characteristics of the cross-sectional movement of the tire carcass, it can be concluded that the shifting cross-sectional movement of the tire carcass is the basic vibration within the carcass. The coupled vibration characteristics for the circumferential 

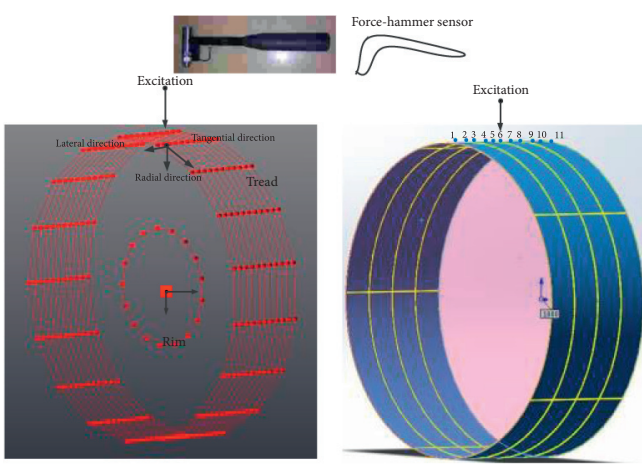

(a)

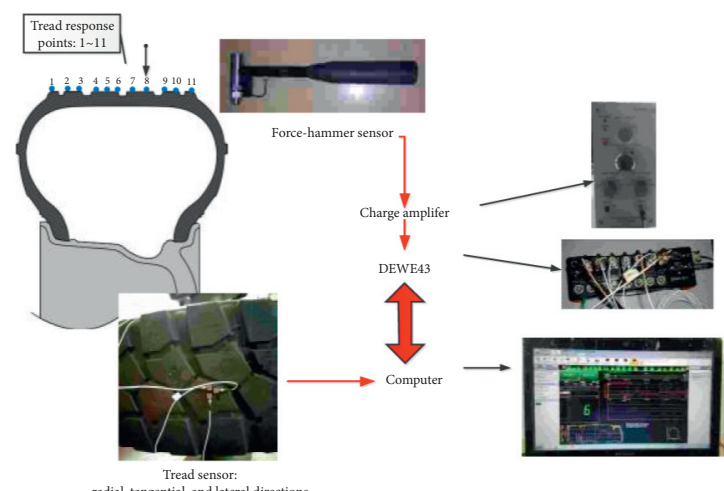

(b)

Figure 3: Modal analysis experiment using the circumferential and cross-sectional features of the carcass: (a) schematic of the analysis; (b) hardware used in the experiment.

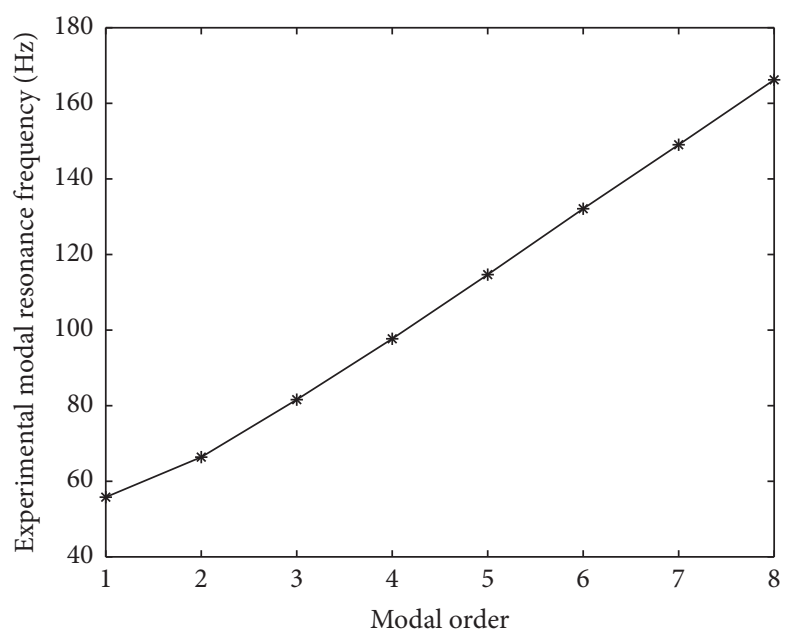

(a)

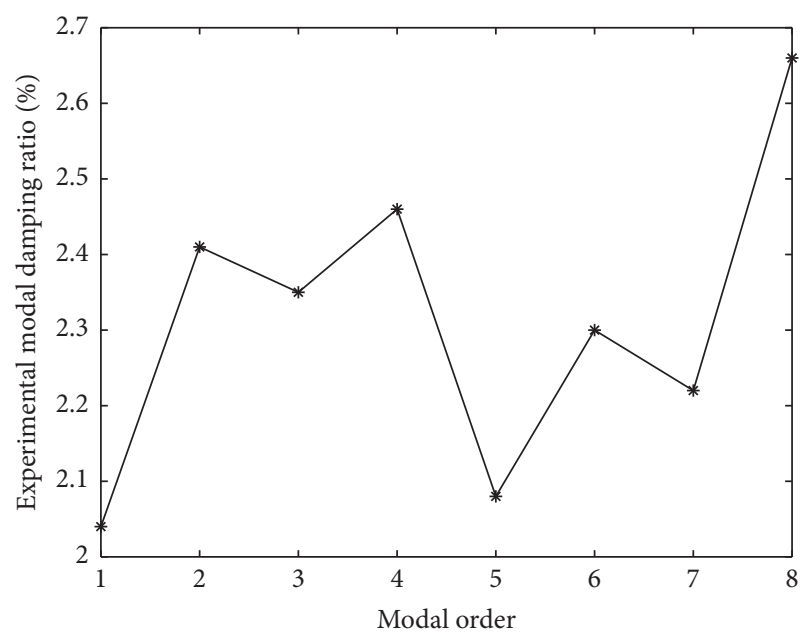

(b)

FIGURE 4: Modal parameters calculated for the first piecewise transfer function: (a) modal resonance frequencies; (b) modal damping ratios.

and shifting cross-sectional vibration of the tire carcass are most pronounced for the modal shape at $160 \mathrm{~Hz}$.

The eight modal resonance frequencies for the second piecewise transfer function are presented in Figure 6, covering the frequency bands between 160 and $280 \mathrm{~Hz}$. The modal coupling shapes for circumferential and crosssectional warping vibration for heavy-load radial tires with a large aspect ratio are shown in Figure 7.

The circumferential vibration for the second piecewise transfer function is consistent with the circumferential vibration for the first transfer function. However, the crosssectional warping vibration for the tire carcass calculated from the second piecewise transfer function $(160-280 \mathrm{~Hz})$ is different from the shifting vibration of the tire carcass calculated from the first piecewise transfer function $(0-160 \mathrm{~Hz})$.

By examining the results for the second piecewise transfer function, it can be concluded that the crosssectional warping vibration of the tire carcass is the basic vibration across frequencies ranging from 160 to $280 \mathrm{~Hz}$.
According to this basic cross-sectional warping vibration, the circumferential vibration of the tire carcass ranges from a shifting modal shape to an eight-sectional modal shape.

As a result of the larger tire damping within the high frequency band $(280-400 \mathrm{~Hz})$, the diagrams for the third piecewise transfer function show instability and the experimental modal parameters for the third piecewise transfer function are difficult to identify.

Another thing to note is that structure-cavity-coupled modal resonance frequencies appear at $120 \mathrm{~Hz}$ (the firstorder structure-cavity resonance frequency), $240 \mathrm{~Hz}$ (the second-order structure-cavity resonance frequency), and $360 \mathrm{~Hz}$ (the third-order structure-cavity resonance frequency). However, the impact of structure-cavitycoupled characteristics on tire vibration is relatively small. So, the structure-cavity coupled properties can be ignored for planar vibration analysis of heavy-load radial tires.

As an additional measure, an MAC matrix was computed (see Figure 8) to validate the orthogonal modal 


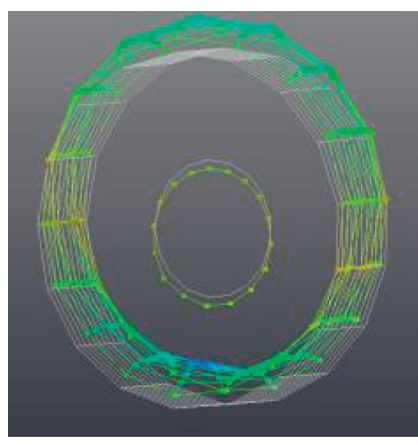

(a)

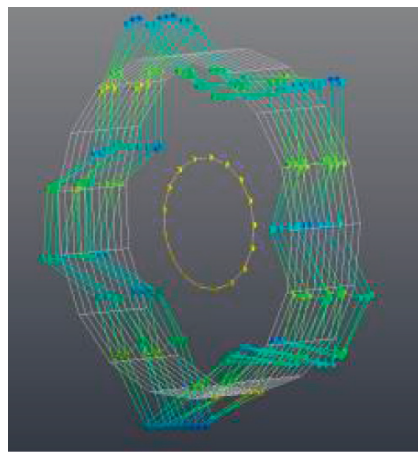

(e)

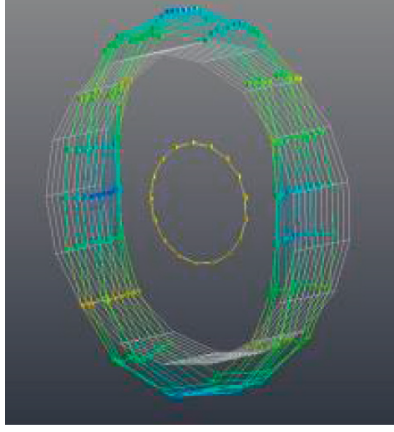

(b)

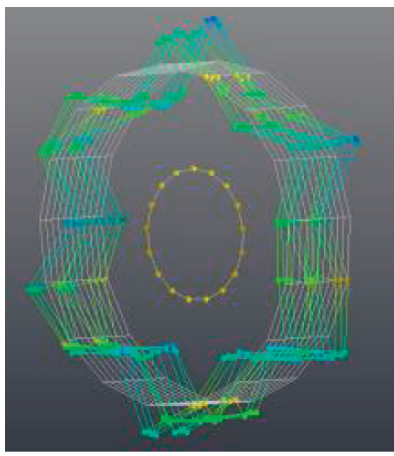

(f)

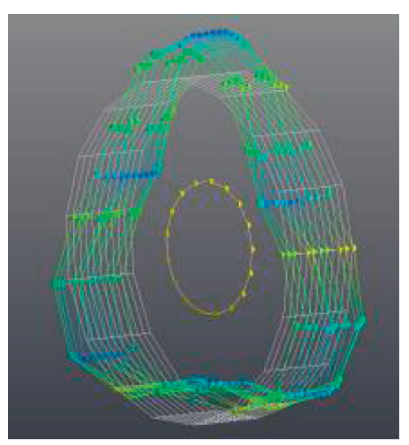

(c)

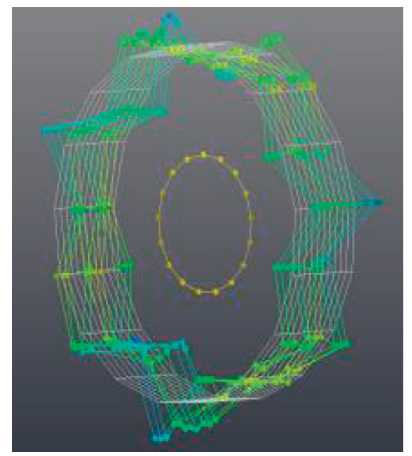

(g)

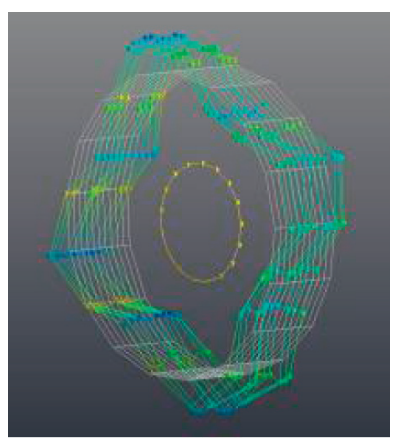

(d)

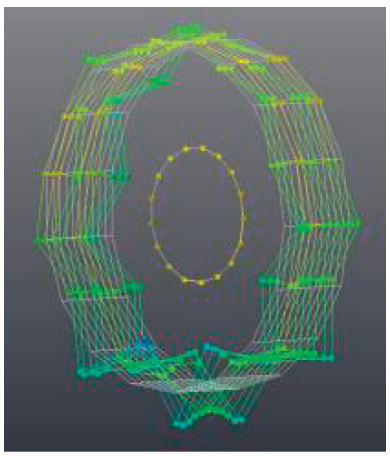

(h)

FiguRE 5: Different modal shapes for the first piecewise transfer function $(0-160 \mathrm{~Hz})$ : (a) shifting shape; (b) two-sectional shape; (c) threesectional shape; (d) four-sectional shape; (e) five-sectional shape; (f) six-sectional shape; (g) seven-sectional shape; (h) eight-sectional shape.

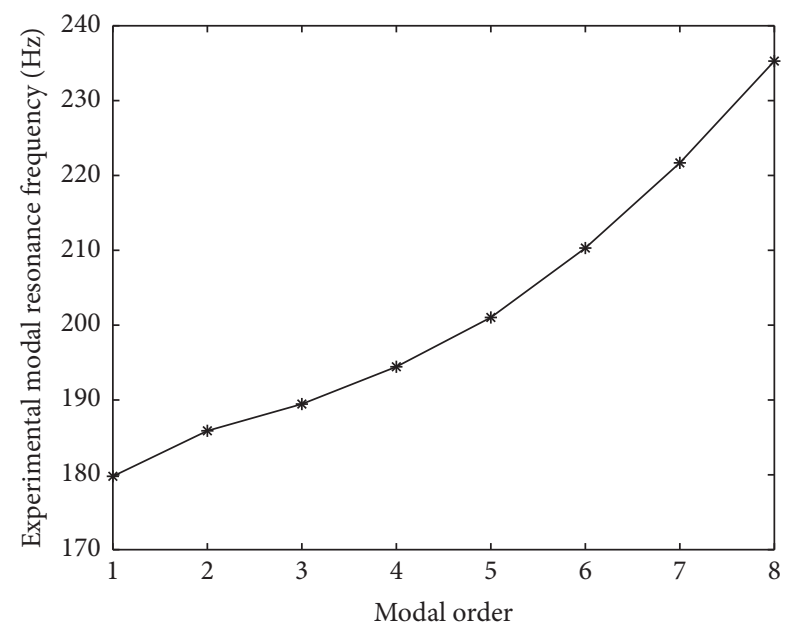

(a)

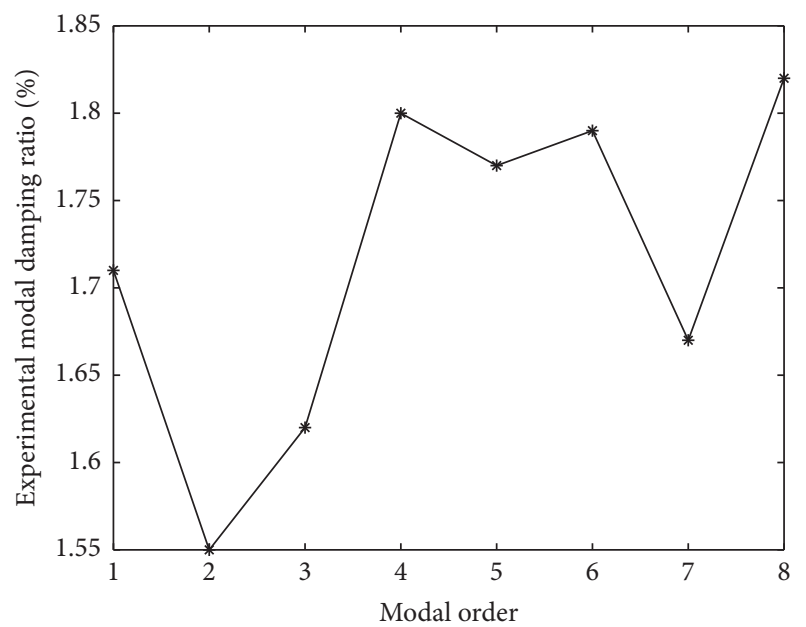

(b)

FIGURE 6: Modal parameters calculated for the second piecewise transfer function: (a) modal resonance frequencies; (b) modal damping ratios.

vectors. The outcome of this assessment was that the MAC values for the modal analysis experiment were less than 0.2 , confirming that the modal vectors were orthogonal.

Taking the coupled characteristics of the circumferential and cross-sectional vibration of tire carcass into consideration, the modal analysis experiment was able to effectively evaluate planar vibration up to $400 \mathrm{~Hz}$. The outcomes of this experiment were as follows:
(1) The planar transfer functions for heavy-load radial tires up to $400 \mathrm{~Hz}$ were divided into three parts: uncovering different cross-sectional vibration features for the tire carcass, including both shifting and warping cross-sectional vibration.

(2) The piecewise modal features of heavy-load radial tires were also shown to consist of circumferential vibration of the flexible carcass with different modal 


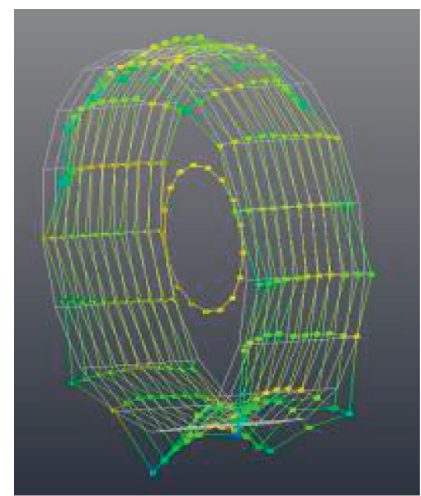

(a)

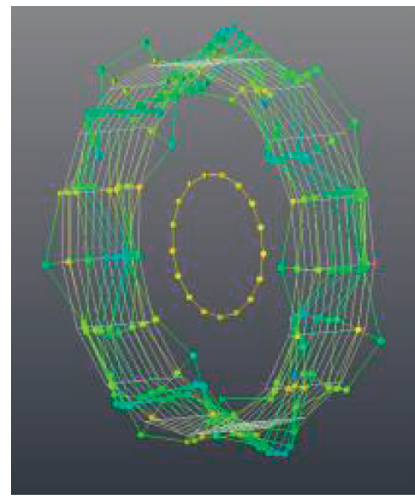

(e)

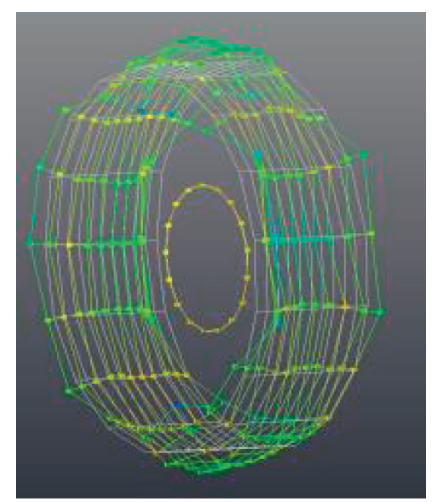

(b)

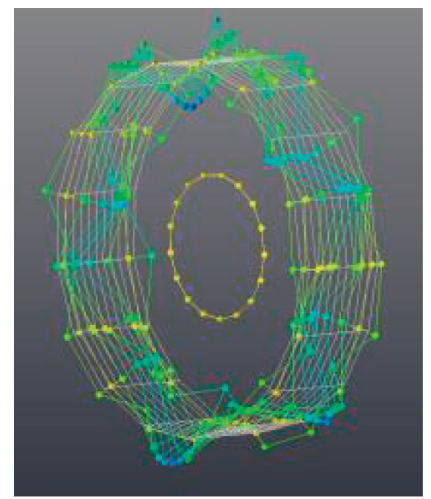

(f)

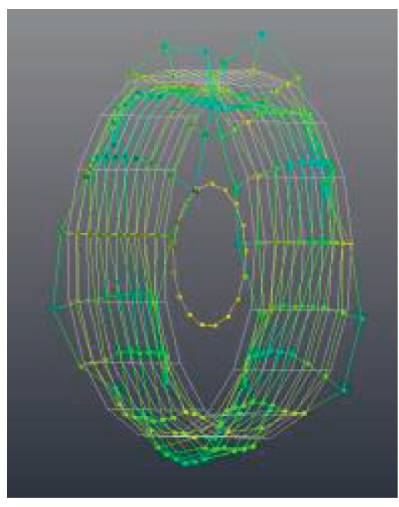

(c)

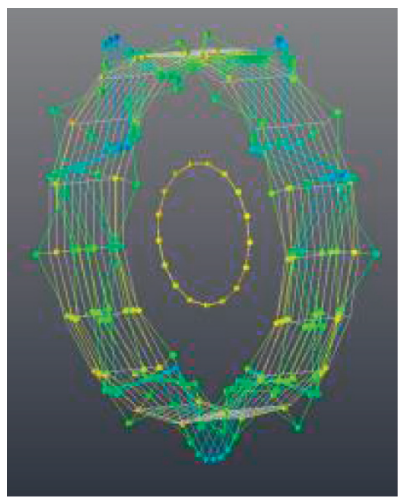

(g)

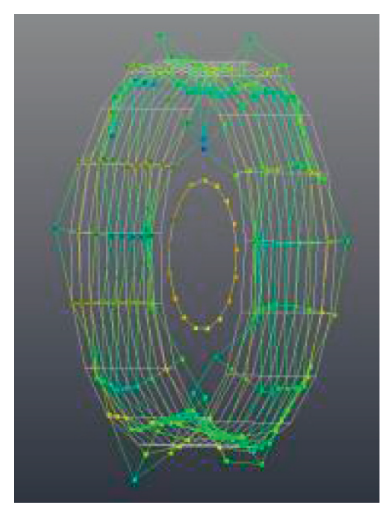

(d)

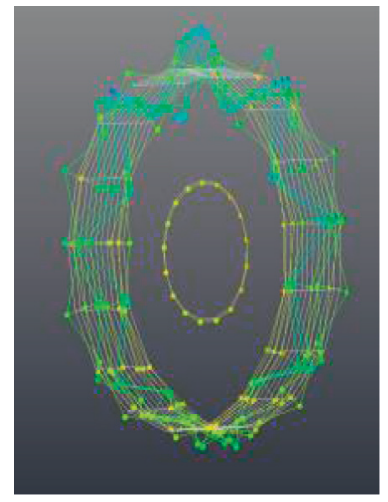

(h)

FIgURE 7: Different modal shapes for the second piecewise transfer function (160-280 Hz): (a) shifting shape; (b) two-sectional shape; (c) three-sectional shape; (d) four-sectional shape; (e) five-sectional shape; (f) six-sectional shape; (g) seven-sectional shape; (h) eightsectional shape.

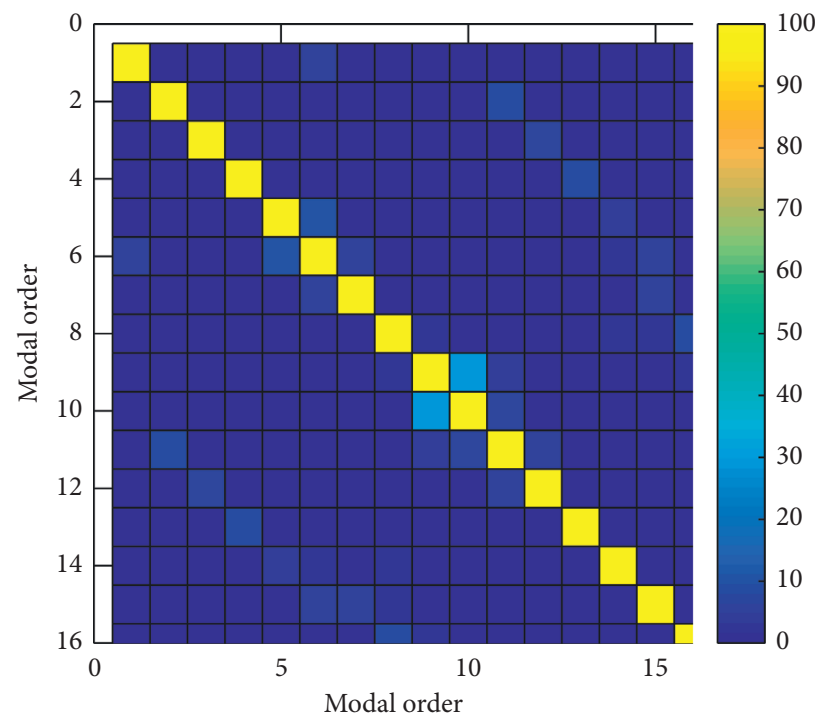

FIGURE 8: MAC matrix.

shapes ranging a shifting movement to an eight-sectional flexible vibration. This circumferential vibration of the flexible carcass is consistent with the flexible bending characteristics of a Euler beam, so applying a flexible beam to the elastic foundation model is able to characterize the piecewise vibration characteristics of heavy-load radial tires with a large aspect ratio.

(3) Damping caused an increased attenuation of vibrations as the frequency increased because of the viscoelastic properties of the carcass rubber. This 


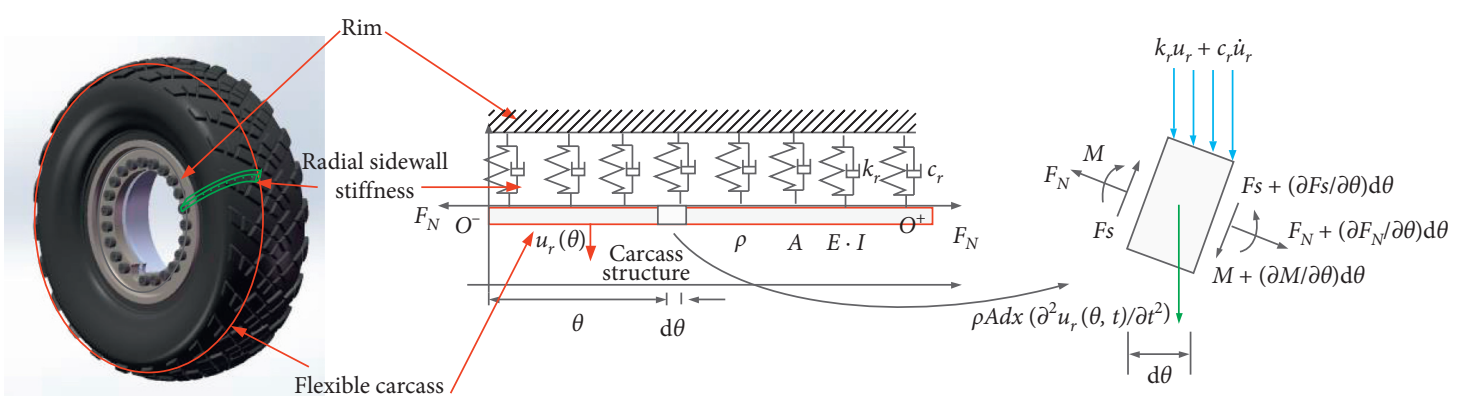

FIgUre 9: Model of the flexible beam on an elastic foundation.

resulted in an unstable modal diagram being produced in the high frequency band.

\section{Model for a Piecewise Flexible Beam on an Elastic Tire Foundation}

3.1. Theoretical Modeling. In this section, we shall be examining the piecewise vibration characteristics of heavyload radial tires and developing a flexible beam on an elastic tire foundation model. The proposed model is based upon the piecewise vibration characteristics resulting from different cross-sectional vibration features already uncovered in the previous section. Some assumptions to be highlighted here are that the bending deformation of the carcass beam is taken to be the main deformation, whilst shear deformation and the moment of inertia for the cross-section can be ignored.

Each aspect of the piecewise planar tire model is illustrated in Figure 9. It consists of two critical elements:

(1) The flexible carcass is simulated as an Euler beam, and inflation is considered to be the axis force of the Euler beam although structural damping of the flexible carcass will also be considered.

(2) The sidewall is regarded as the linear elastic foundation, with proportional damping of the sidewall spring being incorporated to enrich the proposed tire model.

Figure 9 shows the schematic of the model, with $u_{r}$ being the horizontal displacement of the cross-section at time $t$ at some points from the original point of the beam.

The planar kinematic equation for the flexible beam on an elastic foundation model can be expressed as follows:

$$
\frac{E I}{R^{4}} \frac{\partial^{4} u_{r}}{\partial \theta^{4}}+\frac{\eta E I}{R^{4}} \frac{\partial^{5} u_{r}}{\partial \theta^{4} \partial t}+\rho A \frac{\partial^{2} u_{r}}{\partial t^{2}}-\frac{P_{0} b}{R} \frac{\partial^{2} u_{r}}{\partial \theta^{2}}+k_{r} u_{r}+c_{r} u_{r}=0,
$$

where $E I$ is the circumferential bending stiffness of flexible carcass; $R$ is carcass radii and $R=0.65 \mathrm{~m} ; \eta$ is the damping coefficient of structural damping; $\rho \operatorname{Ad} \theta\left(\partial^{2} u_{r} / \partial t^{2}\right)$ is the inertial forces on the microsegment; $F_{N}$ is the pretension axial force of flexible carcass resulting from the inflating pressure and $F_{N}=P_{0} R b$, in which $P_{0}$ is the inflation pressure and $b$ is the carcass width and $b=0.35 \mathrm{~m} ; k_{r}$ is the radial sidewall stiffness; and $c_{r}$ is the radial damping coefficient of sidewall spring.

Derived with the modal expansion method by solving equation (1), the analytical modal resonant frequency $\mathrm{Fre}_{a}$ is given as

$$
\operatorname{Fre}_{a}=\frac{1}{2 \pi} \sqrt{\frac{E I / R^{4} n^{4}+P_{0} b / R n^{2}+k_{r}}{\rho A}} .
$$

3.2. Planar Differential Form for the Proposed Tire Model. In order to simplify the solution procedure, the planar differential form of the proposed tire model can also be derived from equation (1). The difference forms for the second-order and fourth-order are

$$
\left\{\begin{array}{l}
\frac{\partial^{2} u_{r}(x, t)}{\partial \theta^{2}} \cong \frac{u_{r, n+1}-2 u_{r, n}+u_{r, n-1},}{\theta^{2}}, \\
\frac{\partial^{4} u_{r}(x, t)}{\partial \theta^{4}} \cong \frac{u_{r, n+2}-4 u_{r, n+2}+6 u_{r, n}-4 u_{r, n-1}+u_{r, n 22}}{\theta^{4}},
\end{array}\right.
$$

Substituting Equation (3) into Equation (1), the planar differential form of the proposed tire model will now be

$$
\begin{aligned}
& u_{r, n+2} \frac{E I}{R^{4} \theta^{4}}-u_{r, n+1}\left(\frac{4 E I}{R^{4} \theta^{4}}+\frac{P_{0} b}{R}\right)+u_{r, n}\left(\frac{6 E I}{R^{4} \theta^{4}}+k_{r}+\frac{2 P_{0} b}{R}\right) \\
& -u_{r, n-1}\left(\frac{4 E I}{R^{4} \theta^{4}}+\frac{P_{0} b}{R}\right)+u_{r, n+2} \frac{E I}{R^{4} \theta^{4}} \\
& +\rho A \ddot{u}_{\mathrm{r}, \mathrm{n}}+\dot{u}_{r, n+2} \frac{\eta E I}{R^{4} \theta^{4}}-\dot{u}_{r, n+1} \frac{4 \eta E I}{R^{4} \theta^{4}}+\dot{u}_{r, n}\left(\frac{6 \eta E I}{R^{4} \theta^{4}}+c_{r}\right) \\
& -\dot{u}_{r, n-1} \frac{4 \eta E I}{R^{4} \theta^{4}}+\dot{u}_{r, n+2} \frac{\eta E I}{R^{4} \theta^{4}}=0 .
\end{aligned}
$$


The kinematic equations for $N$ carcass segments are combined for the planar second-order difference tire model. This is given in Equation (5). The mass and stiffness matrixes of the planar second-order difference tire model are symmetric. The damping matrix $C$ consists of two parts: the structural damping matrix of the flexible carcass and the proportional damping matrix of the sidewall spring:

$$
M \ddot{U}+C \dot{U}+K U=f(t),
$$

where

$$
U=\left[u_{r, 1} u_{r, 2} \cdots u_{r, n-1} u_{r, n}\right]^{T},
$$$$
M=\left[\begin{array}{lllll}
\rho A & & & & \\
& & & & \\
& \rho A & & & \\
& & \ddots & & \\
& & & \rho A & \\
& & & & \\
& & & & \rho A
\end{array}\right] \text {, }
$$$$
K=\left[\begin{array}{ccccccc}
\alpha_{3} & \alpha_{1} & \alpha_{2} & 0 & \cdots & \alpha_{2} & \alpha_{1} \\
\alpha_{1} & \alpha_{3} & \alpha_{1} & \alpha_{2} & \cdots & 0 & \alpha_{2} \\
\alpha_{2} & \alpha_{1} & \alpha_{3} & \alpha_{1} & \cdots & 0 & 0 \\
0 & \alpha_{2} & \alpha_{1} & \alpha_{3} & \cdots & 0 & 0 \\
\vdots & \vdots & \vdots & \vdots & \ddots & \vdots & \vdots \\
\alpha_{2} & 0 & 0 & 0 & \cdots & \alpha_{3} & \alpha_{1} \\
\alpha_{1} & \alpha_{2} & 0 & 0 & \cdots & \alpha_{1} & \alpha_{3}
\end{array}\right],
$$

$\alpha_{1}=\frac{E I}{R^{4} \theta^{4}}$,

$\alpha_{2}=-\left(\frac{4 E I}{R^{4} \theta^{4}}+\frac{P_{0} b}{R}\right)$,

$\alpha_{3}=\frac{6 E I}{R^{4} \theta^{4}}+k_{r}+\frac{2 P_{0} b}{R}$,

$\alpha_{4}=\alpha_{2}$,

$\alpha_{5}=\alpha_{1}$,

$$
C=\left[\begin{array}{ccccccc}
\beta_{3} & \beta_{1} & \beta_{2} & 0 & \cdots & \beta_{2} & \beta_{1} \\
\beta_{1} & \beta_{3} & \beta_{1} & \beta_{2} & \cdots & 0 & \beta_{2} \\
\beta_{2} & \beta_{1} & \beta_{3} & \beta_{1} & \cdots & 0 & 0 \\
0 & \beta_{2} & \beta_{1} & \beta_{3} & \cdots & 0 & 0 \\
\vdots & \vdots & \vdots & \vdots & \ddots & \vdots & \vdots \\
\beta_{2} & 0 & 0 & 0 & \cdots & \beta_{3} & \beta_{1} \\
\beta_{1} & \beta_{2} & 0 & 0 & \cdots & \beta_{1} & \beta_{3}
\end{array}\right],
$$

$$
\begin{aligned}
& \beta_{1}=\frac{\eta E I}{R^{4} \theta^{4}}, \\
& \beta_{2}=\frac{4 \eta E I}{R^{4} \theta^{4}}, \\
& \beta_{3}=\frac{6 \eta E I}{R^{4} \theta^{4}}+c_{r 1}, \\
& \beta_{4}=\beta_{2}, \\
& \beta_{5}=\beta_{1} .
\end{aligned}
$$

3.3. Analytic Piecewise Transfer Function. The equation for the frequency domain can be transformed from that of the time domain (Equation (5) for the planar second-order difference tire model):

$$
\left(-\omega^{2} M+j \omega C+K\right)\{U(\omega)\}=\{F(\omega)\},
$$

where the displacement transfer matrix of the frequency domain is given as $H_{d}(\omega)$.

$$
H_{d}(\omega)=\left(-\omega^{2} M+j \omega C+K\right)^{-1} .
$$

As the damping matrix $C$ is symmetrical, the matrixes $M$, $C$, and $K$ can be orthogonal with the modal shape matrix, as given in the following equation:

$$
\left\{\begin{array}{l}
\operatorname{diag}\left(M_{i}\right)=\Phi^{T} M \Phi, \\
\operatorname{diag}\left(C_{i}\right)=\Phi^{T} C \Phi, \\
\operatorname{diag}\left(K_{i}\right)=\Phi^{T} K \Phi,
\end{array}\right.
$$

where $\Phi=\left[\left\{\phi_{1}\right\}\left\{\phi_{2}\right\} \ldots\left\{\phi_{N}\right\}\left\{\phi_{N+1}\right\}\left\{\phi_{N+2}\right\} \ldots\left\{\phi_{2 * N}\right\}\right.$ $\left.\left\{\phi_{2 * N+1}\right\}\left\{\phi_{2 * N+2}\right\} \ldots\left\{\phi_{3 * N}\right\}\right]$ is the modal shape matrix; $\left\{\phi_{i}\right\}$ is the vector of $i^{\text {th }}$ modal shape; $\left\{\phi_{1}\right\}-\left\{\phi_{n}\right\}$ are, respectively, the first piecewise modal shapes with the combined characteristic of the circumferential and shifting cross-sectional vibration $(0-160 \mathrm{~Hz}) ;\left\{\phi_{N+1}\right\}-\left\{\phi_{2 * N}\right\}$ are, respectively, the second piecewise modal shapes with the combined characteristic of the circumferential and warping cross-sectional vibration $(160-280 \mathrm{~Hz}) ;\left\{\phi_{2 * N+1}\right\}-\left\{\phi_{3 * N}\right\}$ are, respectively, the third modal shapes with the combined characteristic of the circumferential and high-order bending cross-sectional vibration $(280-400 \mathrm{~Hz})$.

Displacement transfer matrix of frequency domain is calculated as

$$
\begin{aligned}
H_{d}(\omega) & =\sum_{i=1}^{\infty} \frac{\left\{\phi_{i}\right\}\left\{\phi_{i}\right\}^{T}}{M_{i}\left(-\omega^{2}+j^{2} \xi_{i} \omega_{i} \omega+\omega_{i}^{2}\right)} \\
& \approx \sum_{i=1}^{3 * N} \frac{\left\{\phi_{i}\right\}\left\{\phi_{i}\right\}^{T}}{M_{i}\left(-\omega^{2}+j^{2} \xi_{i} \omega_{i} \omega+\omega_{i}^{2}\right)},
\end{aligned}
$$

where the modal resonant frequency and modal damping are, respectively, referred to $\omega_{i}=\sqrt{K_{i} / M_{i}}, \xi_{i}=C_{i} / 2 M_{i} \omega_{i}$. 
Besides, acceleration transfer function of frequency domain is calculated as

$$
H_{a}^{p q}(\omega)=\sum_{i=1}^{3 * N}-\omega^{2} \frac{\phi_{p i} \phi_{q i}}{M_{i}\left(\omega_{i}^{2}-\omega^{2}+j 2 \xi_{i} \omega_{i} \omega\right)} .
$$

Considering the sectional modal feature of the heavyloaded radial tire with a large aspect ratio, the piecewise modal truncation method with the calculated modal order $K$ is utilized and the truncated transfer function is calculated as

$$
H_{a}^{p q}(\omega)=H_{a \text { first }}^{p q}(\omega)+H_{a \text { second }}^{p q}(\omega)+H_{a \text { third }}^{p q}(\omega),
$$

where the piecewise transfer functions are, respectively, presented as

$$
\begin{aligned}
H_{a \text { first }}^{p q}(\omega) & =\sum_{i=1}^{K}-\omega^{2} \frac{\phi_{p i} \phi_{q i}}{M_{i}\left(\omega_{i}^{2}-\omega^{2}+j 2 \xi_{i} \omega_{i} \omega\right)}, \\
H_{a \text { second }}^{p q}(\omega) & =\sum_{i=N+1}^{N+K}-\omega^{2} \frac{\phi_{p i} \phi_{q i}}{M_{i}\left(\omega_{i}^{2}-\omega^{2}+j 2 \xi_{i} \omega_{i} \omega\right)}, \\
H_{a}^{p q} \text { third } & (\omega)=\sum_{i=2 * N+1}^{2 * N+K}-\omega^{2} \frac{\phi_{p i} \phi_{q i}}{M_{i}\left(\omega_{i}^{2}-\omega^{2}+j 2 \xi_{i} \omega_{i} \omega\right)} .
\end{aligned}
$$

The second-order difference equation for the tire model can be given with a freedom of $N^{*} N$, and the piecewise transfer function can then be investigated analytically.

The following observations can be made about the proposed tire model:

(1) The stiffness matrix of the planar second-order difference tire model consists of three equivalent stiffness parameters 1,2 , and 3 . These stiffness parameters are related to the geometric and structural parameters of heavy-load radial tires with a large aspect ratio.

(2) The mass matrix of the planar second-order difference tire model is related to the equivalent mass parameter $A$.

(3) The analytic piecewise transfer function is consistent with the piecewise characteristics uncovered in the modal experiment and the piecewise transfer function experiment.

(4) The model parameters for the planar second-order difference tire model need to be identified separately, according to the piecewise modal resonance frequencies or the piecewise transfer function.

\section{Structural Parameter Identification}

The structural parameters $E I, \rho A$, and $k_{r}$ and damping coefficients $\eta$ and $c_{r}$ of the piecewise flexible beam on an elastic foundation model can be identified using the genetic algorithm (GA). Combining survival of the fittest rules and an exchange mechanism for random chromosome information, GA is considered to be the most efficient global optimization search algorithm.

4.1. Implementation of the Identification Approach. The experimental modal parameters for the first and second piecewise transfer functions were originally identified in Figures 4-7. However, as a result of the greater amount of tire damping within the higher frequency band, the experimental modal parameters for the third piecewise transfer function were difficult to identify. Structural parameter identification can be undertaken on the basis of the following two conditions:

(1) The modal parameters for the first and second piecewise transfer functions were identified clearly in Figures 4 and 6, so the structural parameters EI, $\rho A$, and $k_{r}$ for the first and second piecewise transfer functions can be identified with the error between the analytic modal resonance and the experimental modal frequencies being taken to be the object value. In addition, the damping coefficients $\eta$ and $c_{r}$ can be identified by taking the error between the analytic transfer function and the experimental modal frequencies to be the object value.

(2) Taking the relative error between the analytic transfer functions and the experimental modal frequencies as the object value, the structural parameters $E I, \rho A$, and $k_{r}$ and the damping coefficients $\eta$ and $c_{r}$ can be identified.

\subsection{Optimization Procedure}

4.2.1. Structural Parameter Identification for the First Piecewise Transfer Function. The structural parameters EI, $\rho A$, and $k_{r}$ for the first piecewise transfer function can be identified by using GA and the error between the analytic modal resonance frequencies (Equation (2)) and the experimental modal frequencies (Figure 4). The object value and the identified structural parameters for the first piecewise transfer function are shown in Figure 10. The object value (Figure 10(a)) converges to a steady point when the optimization procedure has reached 30 generations, and the minimum object value is 0.0152 . The identified structural parameters $E I, \rho A$, and $k_{r}$ also converge to a steady value, with the identified results being, respectively, $305.50 \mathrm{~N} / \mathrm{m}$, $36.46 \mathrm{Kg} / \mathrm{m}$, and $4.83 \mathrm{e} 6 \mathrm{~N} / \mathrm{m}$.

Aside from this, by taking the relative error between the analytic piecewise transfer function and the experimental transfer function as the object value, the damping coefficients $\eta$ and $c_{r}$ can be identified. The identification procedure for the first piecewise transfer function is shown in Figure 11. The object value (Figure 11(a)) converges to a steady point as the optimization procedure reaches 22 generations, and the minimum object value is 0.0109 . Note also that the identified damping coefficients $\eta$ and $c_{r}$ converge to a steady value, with the identified results being, respectively, $4 e-4$ and $1 e-4$. 

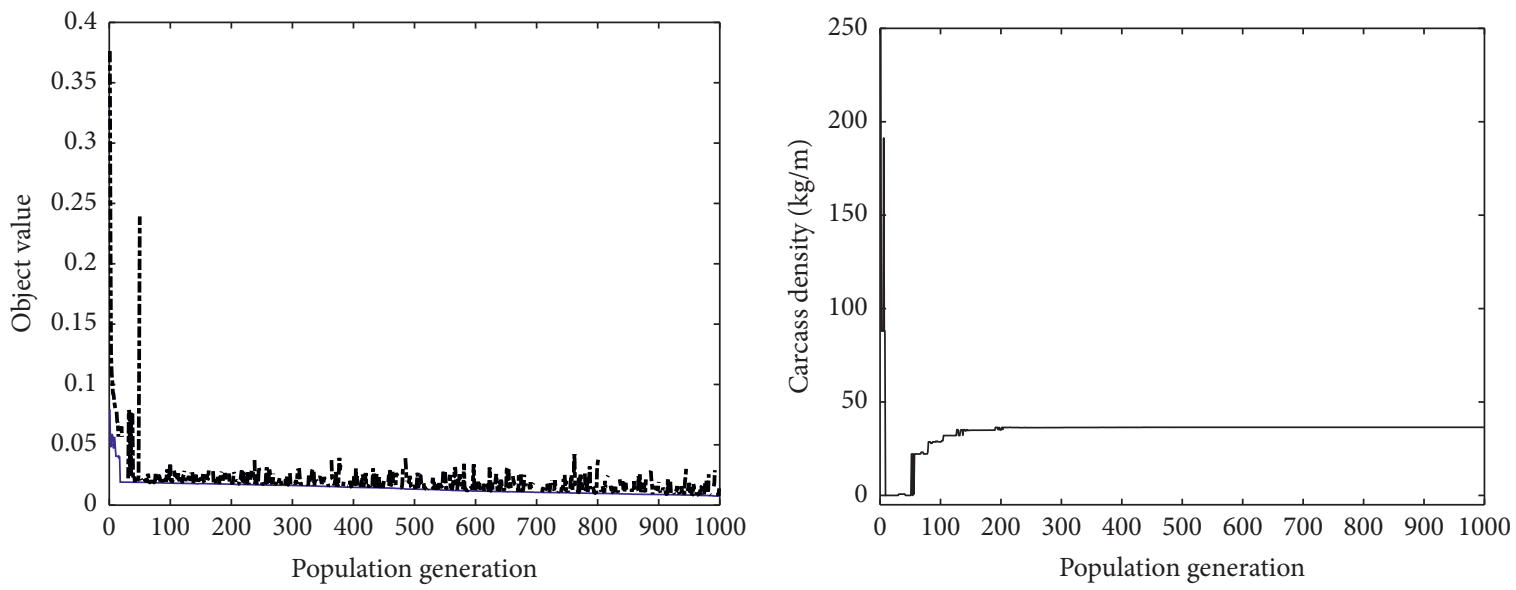

- Best value

---. Average value

(a)

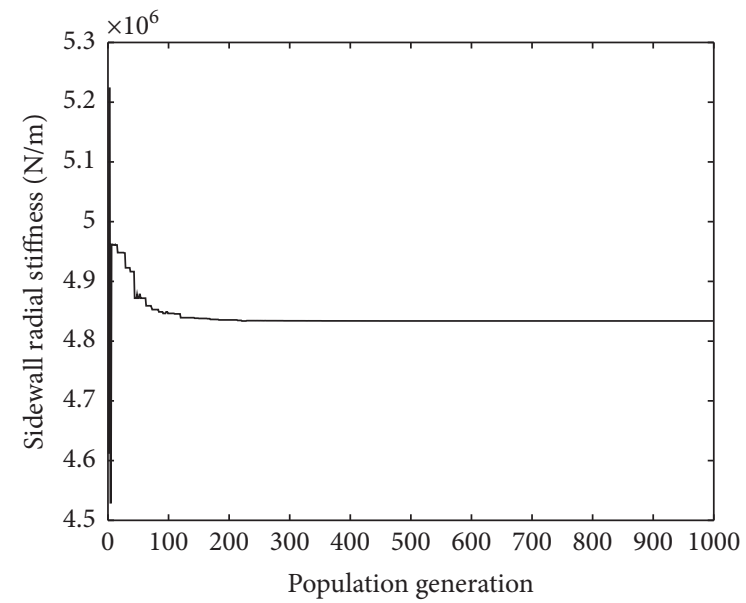

(c)

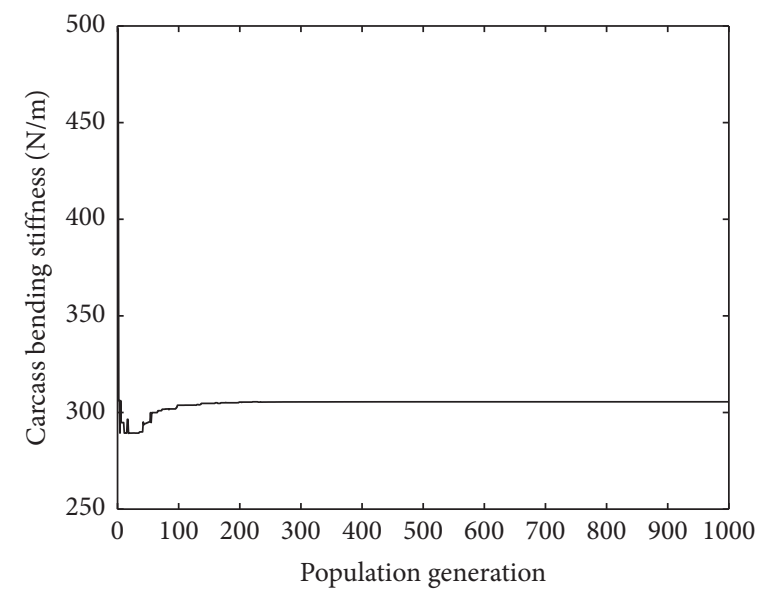

(d)

FiguRE 10: Structural parameters for the first piecewise transfer function: (a) object values for the identification procedure; (b) carcass density for the identification procedure; (c) sidewall radial stiffness for the identification procedure; (d) carcass bending stiffness for the identification procedure.
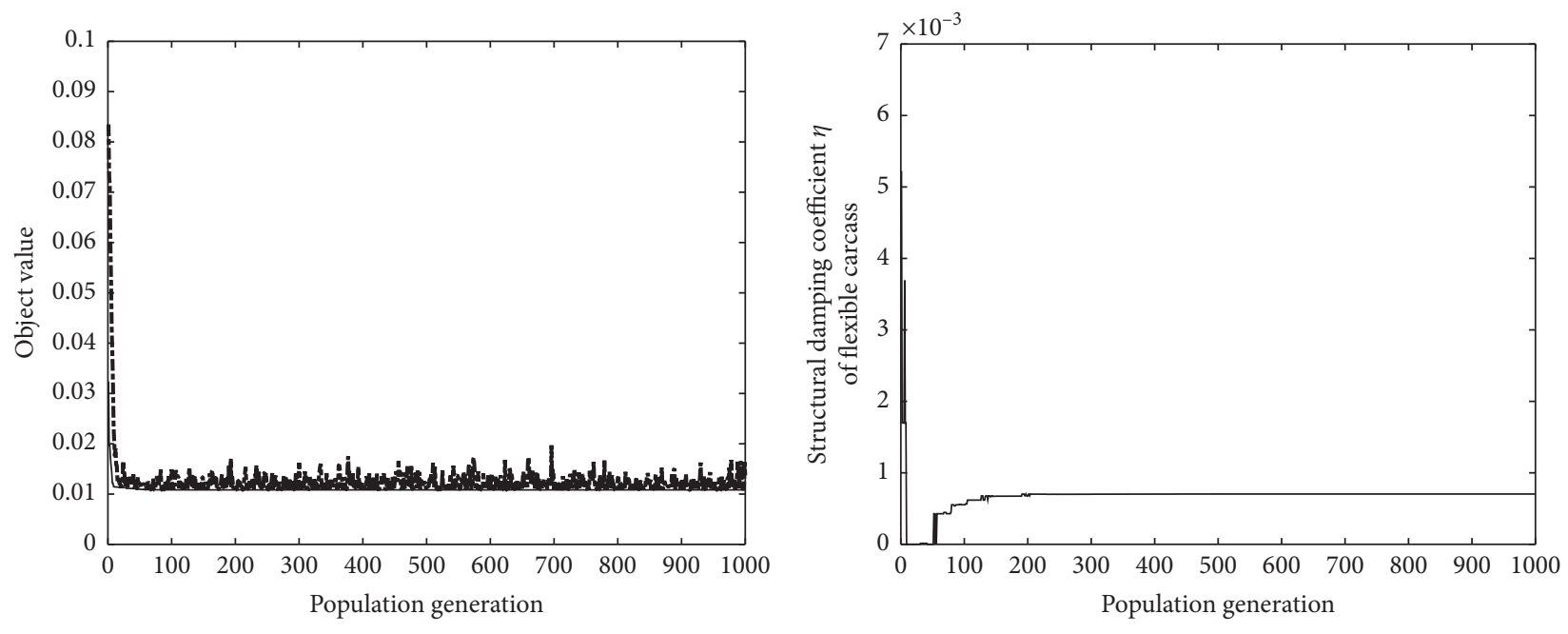

-..- Average value 


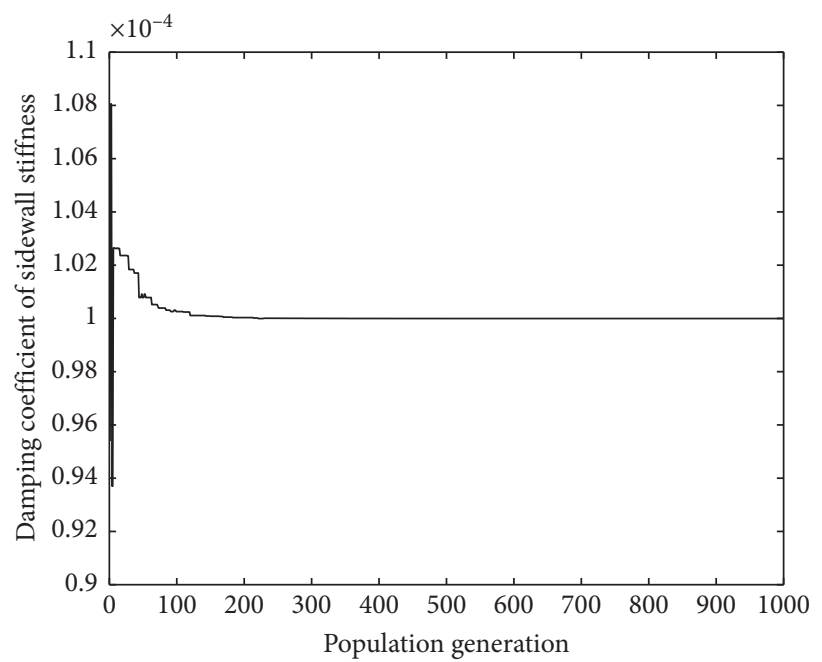

(c)

Figure 11: Damping coefficients for the first piecewise transfer function: (a) object values for the identification procedure; (b) structural damping of the flexible carcass for the identification procedure; (c) proportional damping of the sidewall for the identification procedure.

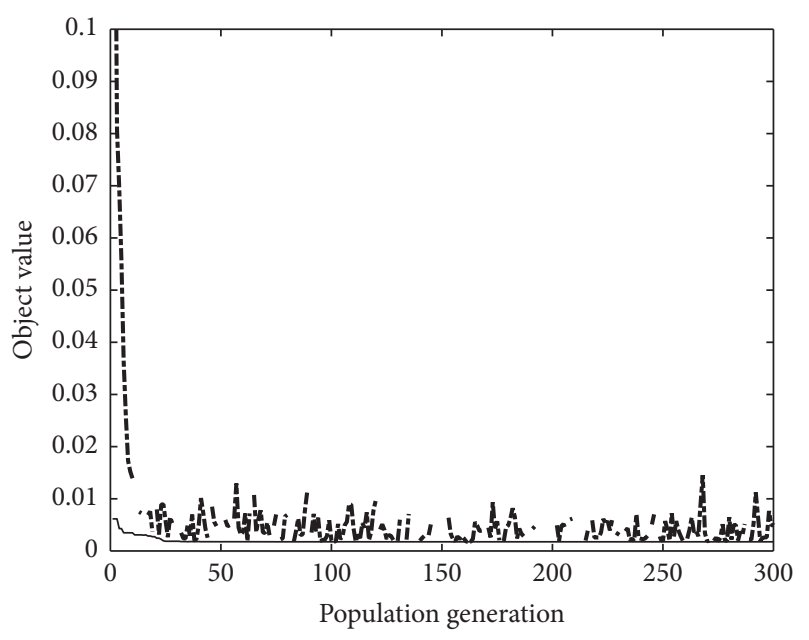

- Best value

---- Average value

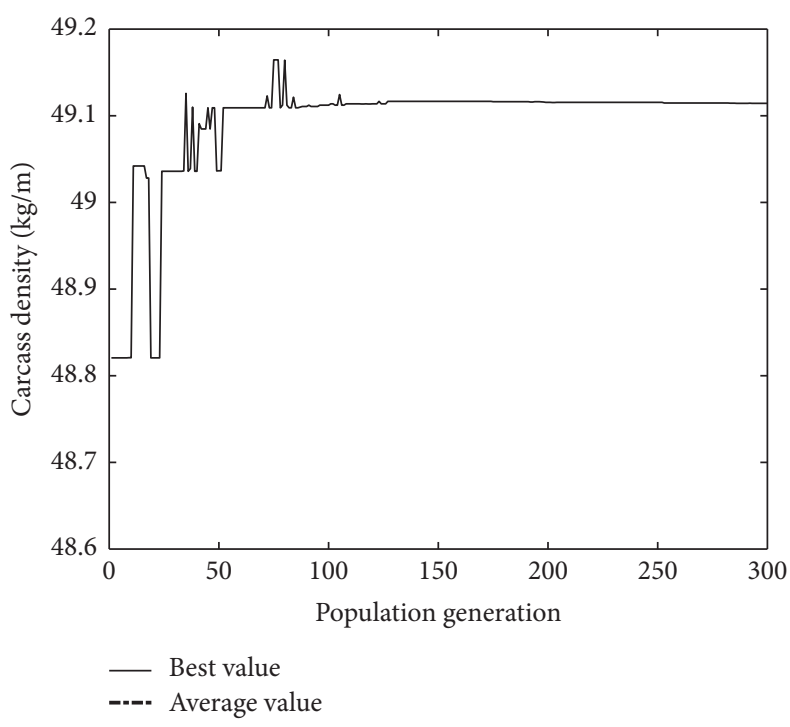

(b)

FIGURE 12: Continued. 


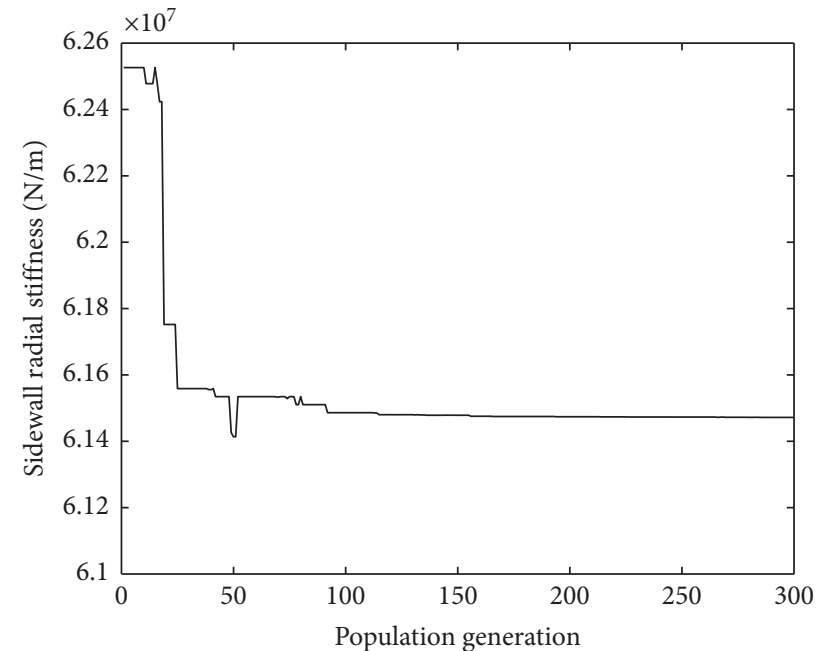

- Best value

---- Average value

(c)

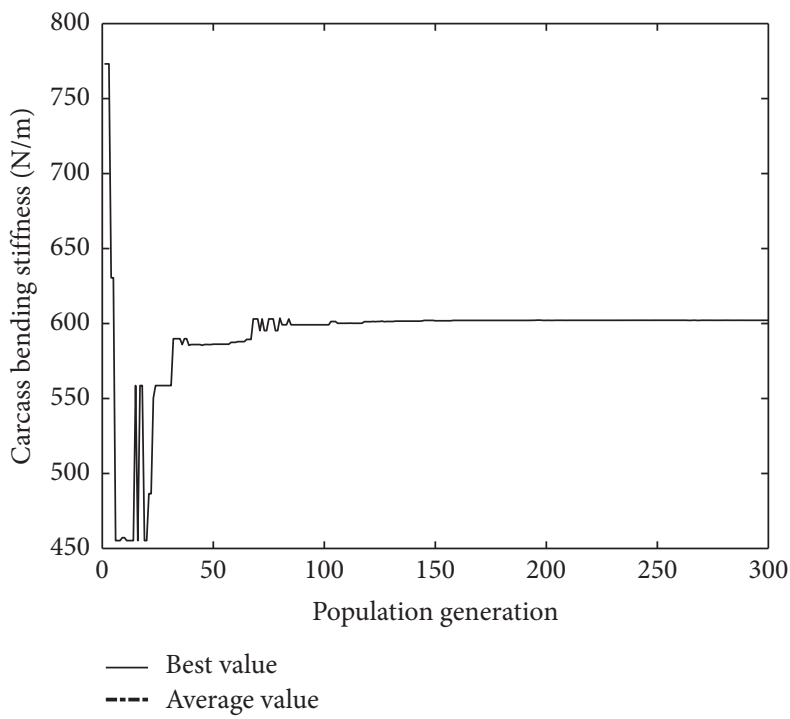

(d)

FiguRE 12: Structural parameters for the second piecewise transfer function: (a) object values for the identification procedure; (b) carcass density for the identification procedure; (c) sidewall radial stiffness for the identification procedure; (d) carcass bending stiffness for the identification procedure.

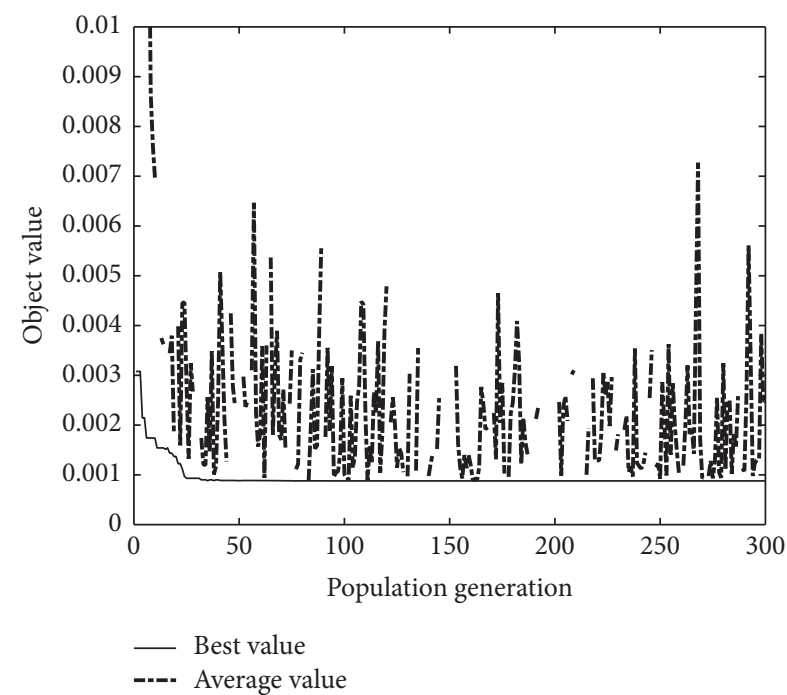

(a)

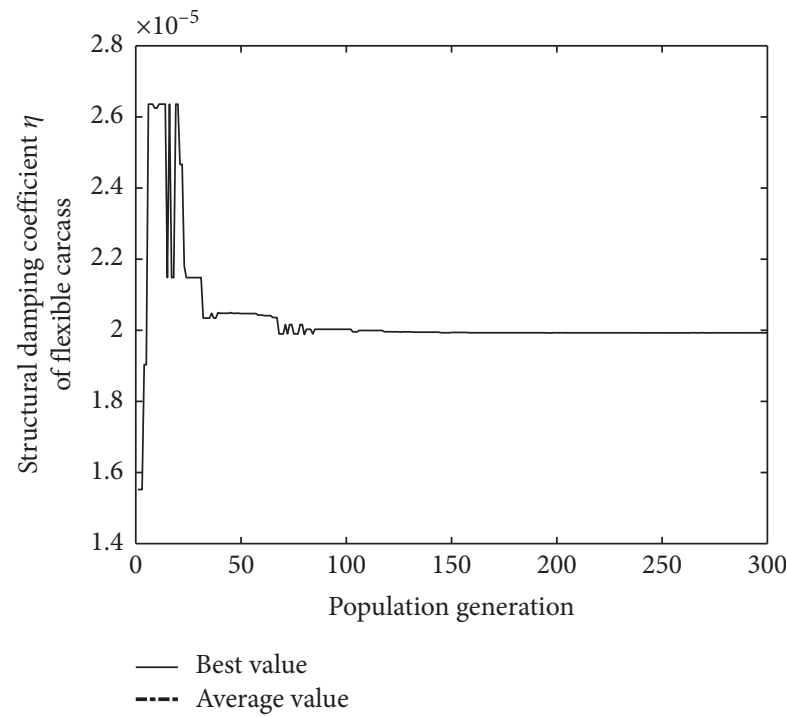

(b)

Figure 13: Continued. 


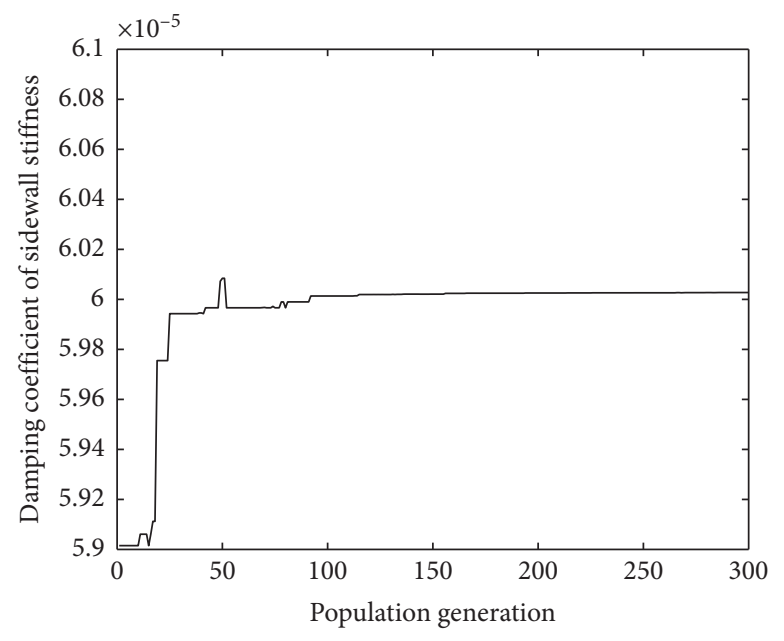

- Best value

---. Average value

(c)

FIGURE 13: Damping coefficients for the second piecewise transfer function: (a) object value for the identification procedure; (b) structural damping of the flexible carcass for the identification procedure; (c) proportional damping of the sidewall for the identification procedure.

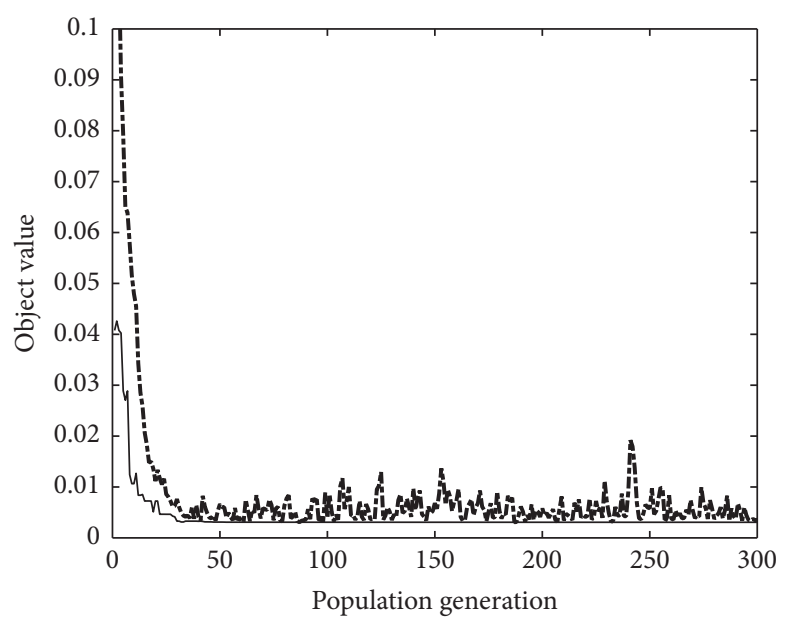

- Best value

---- Average value

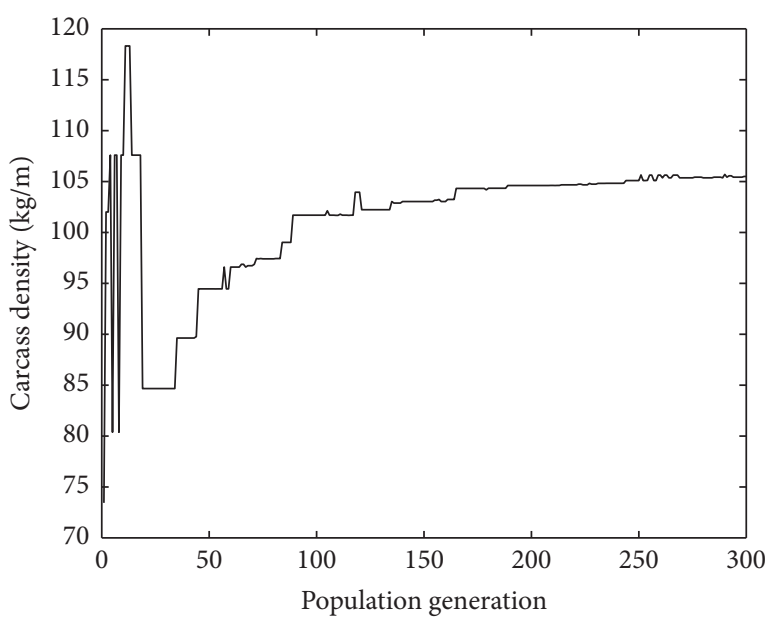

Best value

---- Average value

(a)

(b)

FIgURE 14: Continued. 


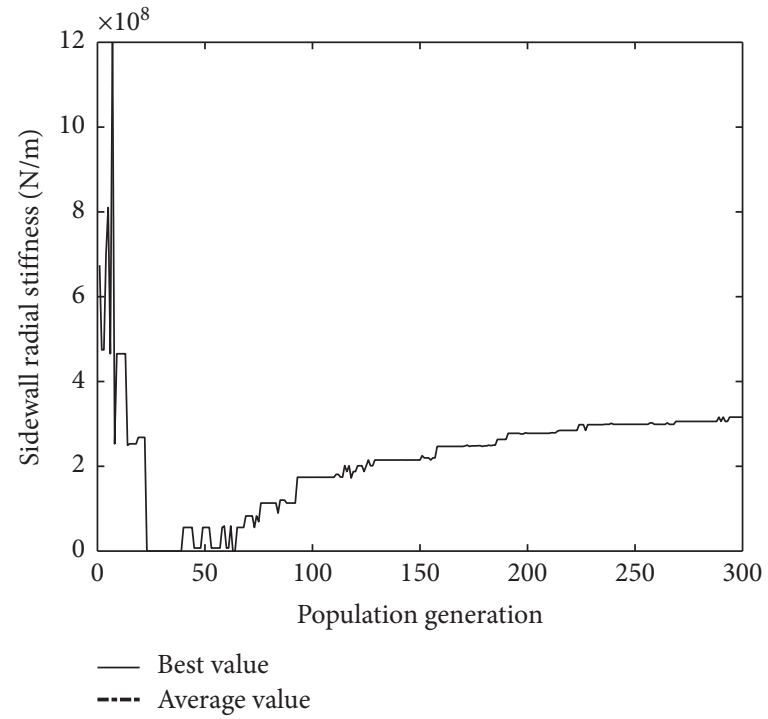

(c)

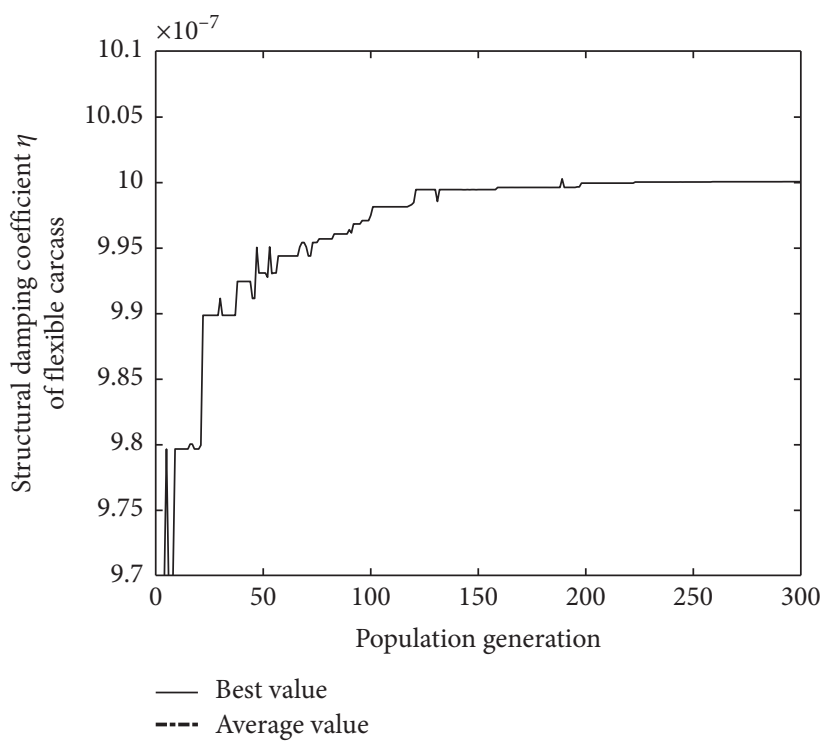

(e)

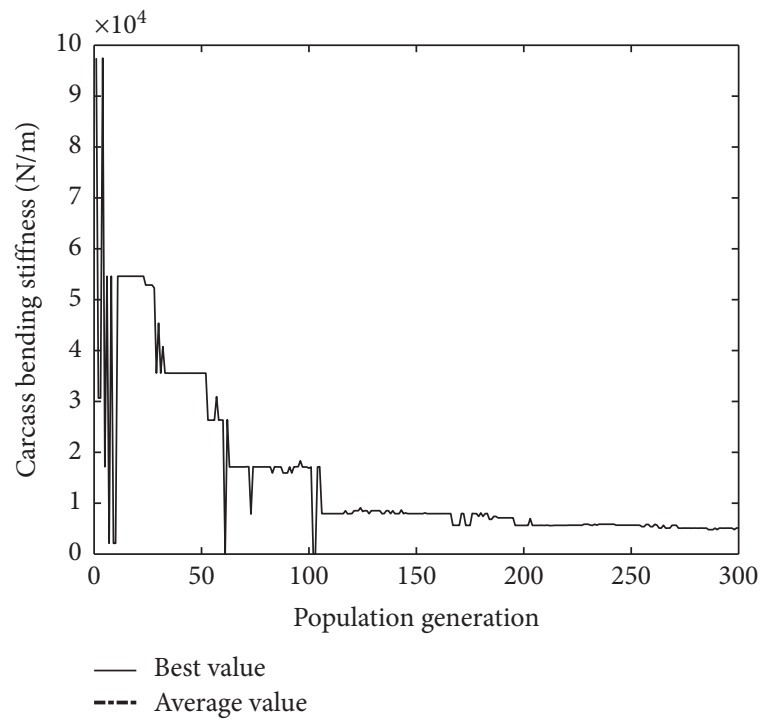

(d)

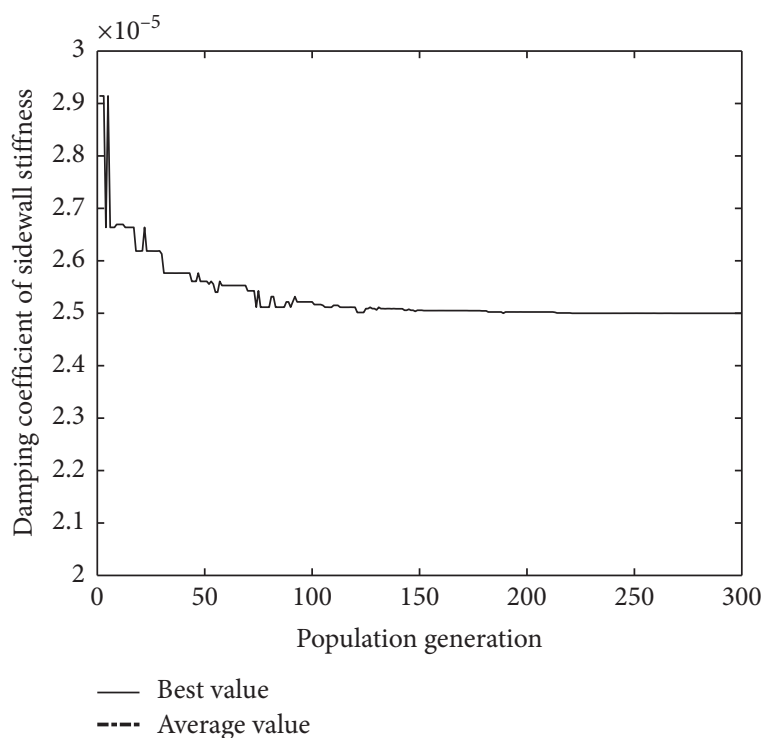

(f)

FIgURE 14: Identified results for the third piecewise transfer function: (a) object values for the identification procedure; (b) carcass density for the identification procedure; (c) sidewall radial stiffness for the identification procedure; (d) carcass bending stiffness for the identification procedure; (e) structural damping of the flexible carcass for the identification procedure; (f) proportional damping of the sidewall for the identification procedure.

4.2.2. Structural Parameter Identification for the Second Piecewise Transfer Function. The structural parameters EI, $\rho A$, and $k_{r}$ for the second piecewise transfer function can also be identified using GA, on the basis of the error between the analytic modal resonance frequencies (Equation (2)) and the experimental modal frequencies (Figure 6). The identification procedure for the second piecewise transfer function is shown in Figure 12. When the optimization procedure has exceeded 30 generations, the object value (Figure 12(a)) converges to a steady point, and the minimum object value is 0.002 . The identified structural parameters $E I, \rho A$, and $k_{r}$ also converge to a steady value, with the identified results being, respectively, $1663.12 \mathrm{~N} / \mathrm{m}$, $77.71 \mathrm{Kg} / \mathrm{m}$, and $9.93 \mathrm{e} 7 \mathrm{~N} / \mathrm{m}$.

In addition, the damping coefficients $\eta$ and $c_{r}$ are identified utilizing GA with the error between the analytical piecewise transfer function and the experimental transfer function. The identification procedure is shown in Figure 13. With the optimization procedure proceeding to 22 generations, the object value (Figure 13(a)) is convergent to the steady point and the minimum value of object value is 0.0098; furthermore, the identified damping coefficients $\eta$ and $c_{r}$ converge to the steady value and the identified results are, respectively, $2.1 e-5$ and $6.1 e-5$. 


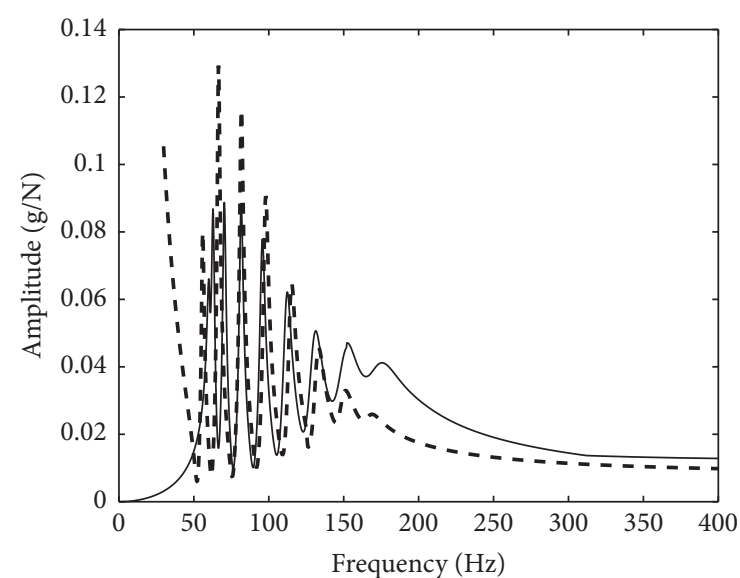

- Computational transfer function with rigid-elastic-coupled tire model

- - - Synthetic transfer function with experimental modal parameters

(a)

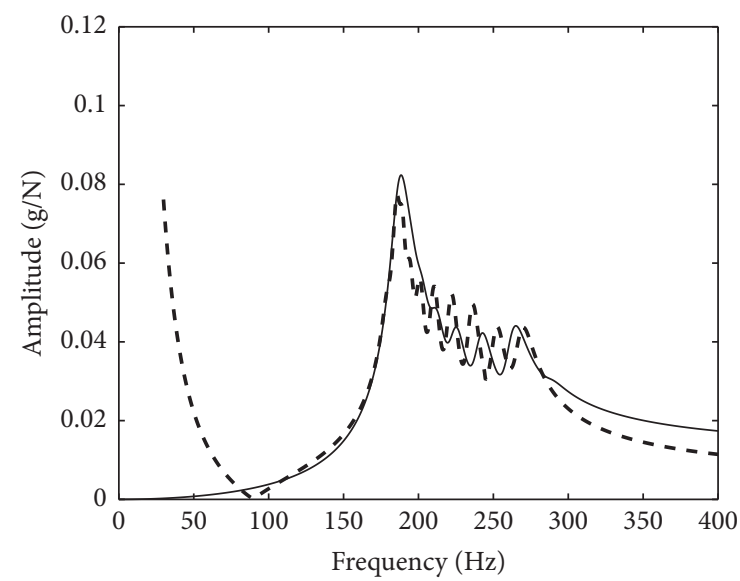

- Computational transfer function with rigid-elastic-coupled tire model

- - - Synthetic transfer function with experimental modal parameters

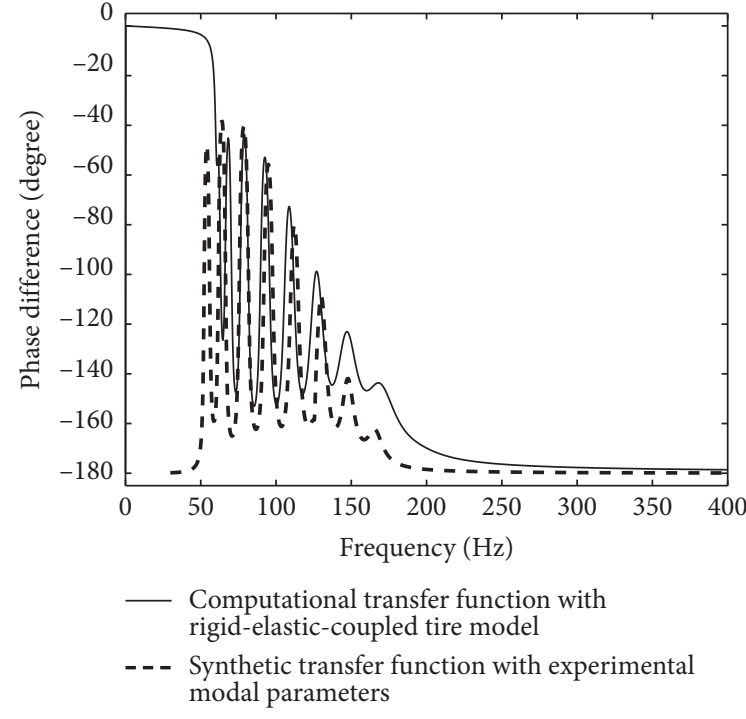

(b)

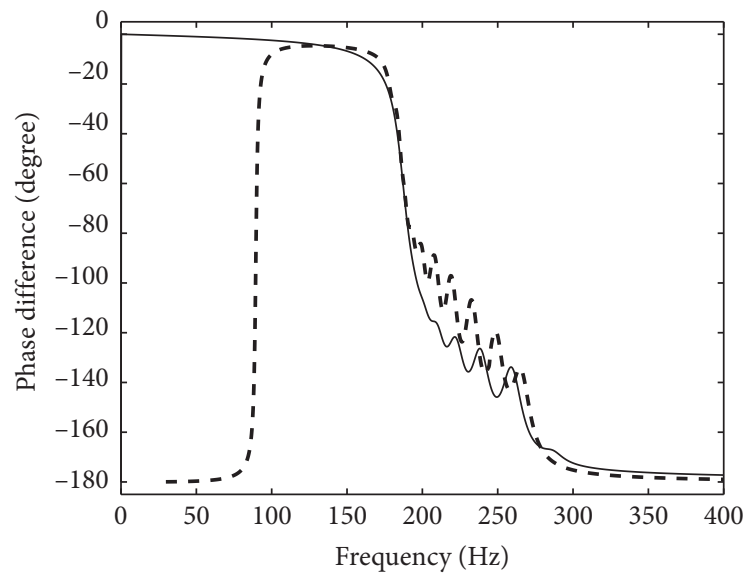

Computational transfer function with rigid-elastic-coupled tire model

- - - Synthetic transfer function with experimental modal parameters

(c)

(d)

Figure 15: Continued. 


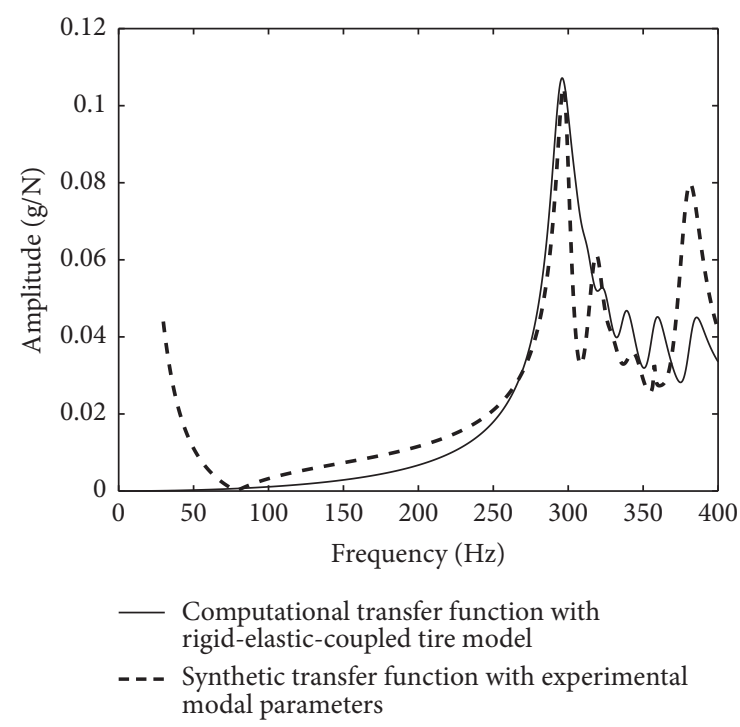

(e)

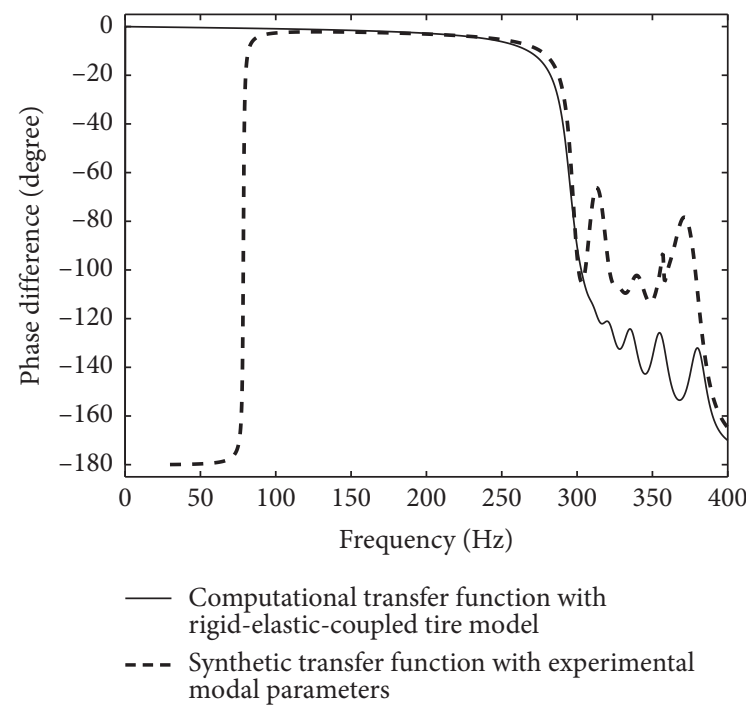

(f)

Figure 15: The piecewise transfer function for a heavy-load radial tire: (a) amplitude of the first piecewise transfer function $(0-160 \mathrm{~Hz})$; (b) phase of the first piecewise transfer function $(0-160 \mathrm{~Hz})$; (c) amplitude of the second piecewise transfer function $(160-280 \mathrm{~Hz})$; (d) phase of the second piecewise transfer function $(160-280 \mathrm{~Hz})$; (e) amplitude of the third piecewise transfer function $(280-400 \mathrm{~Hz})$; (f) phase of the third piecewise transfer function $(280-400 \mathrm{~Hz})$.

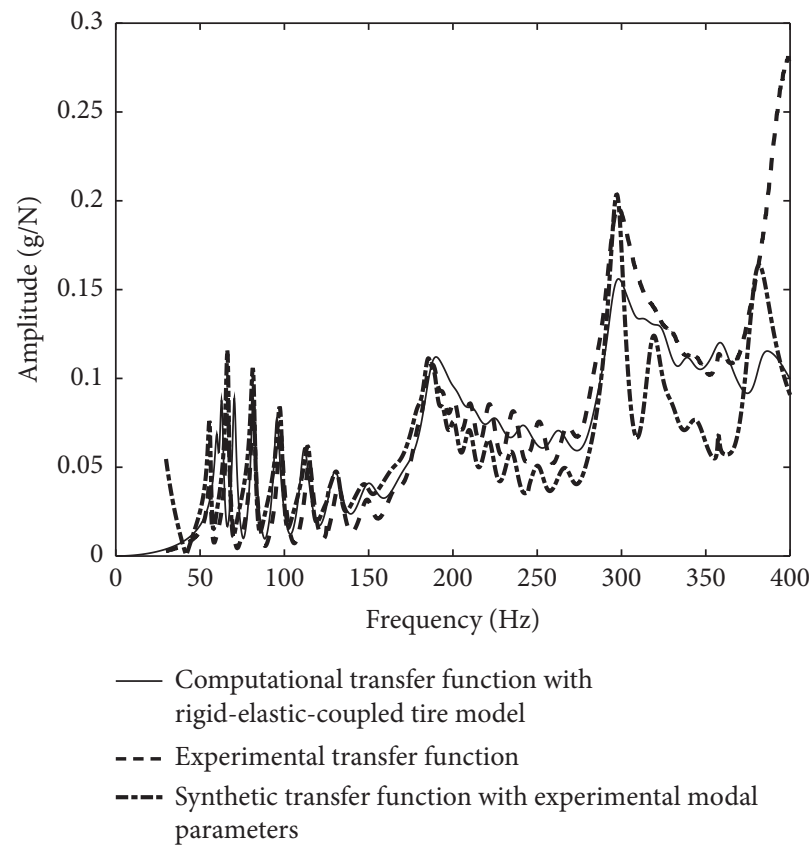

(a)

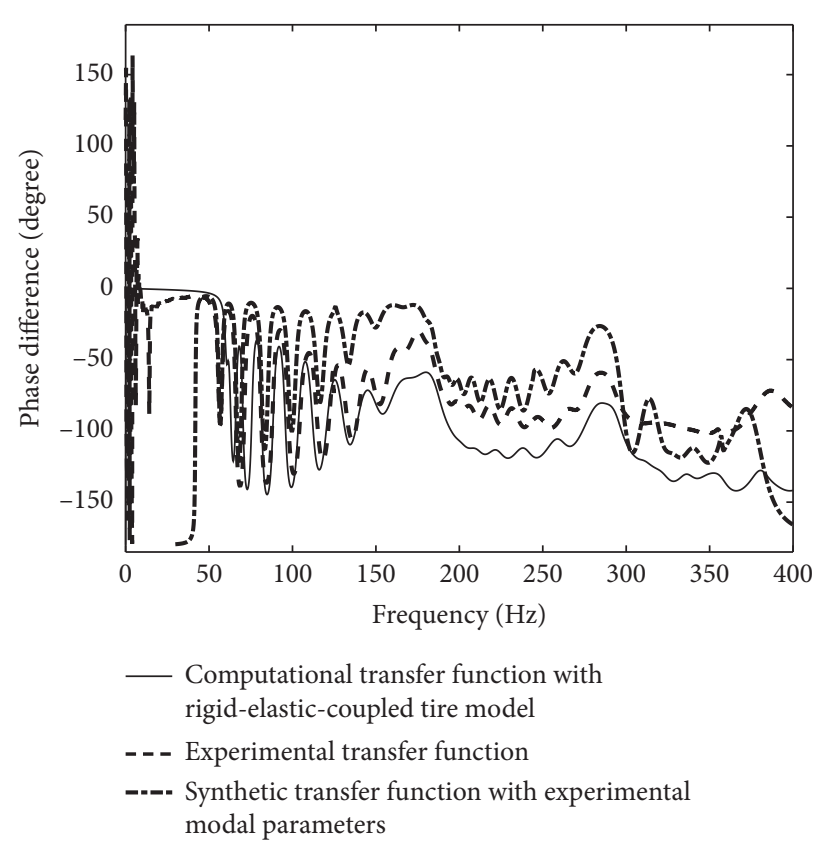

(b)

FIgURE 16: The planar transfer function for point A: (a) amplitude of the transfer function; (b) phase of the transfer function. 
4.2.3. Structural Parameter Identification for the Third Piecewise Transfer Function. Taking the relative error between the analytic piecewise transfer function and the experimental transfer function as the object value, the structural parameters $E I, \rho A$, and $k r$ and damping coefficients $\eta$ and $c_{r}$ for the third piecewise transfer function can again be identified using GA. The identification procedure and results are shown in Figure 14. As the optimization procedure exceeds 33 generations, the object value (Figure 14(a)) converges to a steady point and the minimum object value is 0.0043 . The identified structural parameters $E I, \rho A$, and $k_{r}$ also converge to a steady value, and the identified results are, respectively, $3396.60 \mathrm{~N} / \mathrm{m}$, $106.26 \mathrm{Kg} / \mathrm{m}$, and $3.38 \mathrm{e} 8 \mathrm{~N} / \mathrm{m}$. Note that the identified damping coefficients and $c_{r}$ converge to a steady value as well, with their identified results being, respectively, $1.2 e-6$ and $2.5 e-5$.

\section{Analysis of Planar Vibration Characteristics}

We will now look more closely at the piecewise transfer functions calculated using Equations (15)-(17) and the planar transfer function using Equation (14).

\subsection{Analysis of the Planar Piecewise Transfer Function.} The piecewise transfer functions for the first, second, and third frequency bands were calculated using Equations (15)-(17) and are also shown in Figure 15. If we compare the analytic piecewise transfer functions with the synthetic transfer functions and the experimental piecewise modal parameters, we can see, looking back to Figure 14, that the first, second, and third piecewise transfer functions calculated using the planar second-order difference tire model and the piecewise structural parameters fit well with the synthetic transfer function based on the experimental modal parameters. This validates the piecewise rigid-elastic coupled tire model and the structural parameters we have identified.

5.2. Analysis of the Planar Transfer Function. By means of measuring the response of heavy-load radial tires excited with a hammer, the frequency transfer function can be obtained and compared with the analytic transfer function calculated using the planar second-order difference tire model. The analytic transfer function (Equation (14)) is shown in Figure 16.

The analytic transfer functions for the excitation and response point A (Figure 1(b)) can be compared to the experimental transfer function and the synthetic transfer function that used the experimental modal parameters.

On this basis, the sectional planar transfer function arising from the combined characteristics of circumferential and cross-sectional vibration of the tire carcass can be seen to be consistent with the piecewise flexible beam on an elastic tire foundation model. The proposed model is thus able to characterize the planar vibration of heavy-load radial tires with a large aspect ratio for frequencies up to $400 \mathrm{~Hz}$. Additionally, the analytic transfer functions, identified structural parameters, and damping coefficients all fit well with the experimental transfer function, validating the effectiveness of the identification procedure and the proposed model.

\section{Conclusions}

The planar piecewise vibration characteristics of heavy-load radial tires with a large aspect ratio were investigated using a modal analysis experiment and a piecewise flexible beam on an elastic tire foundation model.

The main outcomes were as follows:

(1) A planar vibration modal for heavy-load radial tires with a large aspect ratio was investigated using an experimental modal method that looked at the combined characteristics of circumferential and cross-sectional vibration. We also examined the piecewise modal characteristics of cross-sectional shifting, warping, and higher-order bending vibration.

(2) In a manner consistent with the modal analysis of piecewise vibration, a piecewise flexible beam on an elastic tire foundation model was proposed and a theoretical model and GA-based parameter identification were employed to investigate the effectiveness of the proposed model.

(3) We discussed the reproducibility of frequency response functions calculated using the proposed tire model and validated it for experimental frequency response functions below $400 \mathrm{~Hz}$.

To conclude,

(1) The sectional frequency response function for heavyload radial tires results from the cross-sectional vibration of the tire carcass. The piecewise frequency response function, however, is mainly influenced by the circumferential vibration of the flexible carcass.

(2) The error between the analytic modal resonance frequencies and the experimental modal resonance frequencies, together with the error between the analytic transfer function and the experimental transfer function, enables the structural parameters and damping coefficients to be identified using a genetic algorithm (GA). These identified parameters have been validated in this paper on the basis of experimental results.

(3) The cross-section vibration of the heavy-load radial tire would be researched with the tire plate model in the further study.

\section{Data Availability}

The data used to support the findings of this study are included within the article.

\section{Conflicts of Interest}

The authors declare that there are no conflicts of interest. 


\section{Acknowledgments}

The research reported here is cosupported by the National Natural Science Foundation of China (Grant no. 51905541), Natural Science Basic Research Plan in Shaanxi province of China (Grant no. 2020JQ487), and Young Talent Fund of University Association for Science and Technology in Shaanxi, China (Grant no. 20190412).

\section{References}

[1] Y. Wei, C. Oertel, X. Li, and L. Yu, "A theoretical model for the tread slip and the effective rolling radius of the tyres in free rolling," Proceedings of the Institution of Mechanical Engineers, Part D: Journal of Automobile Engineering, vol. 231, no. 11, pp. 1461-1470, 2017.

[2] E. O. Bolarinwa, U. Mahadevaiah, D. Marzougui, and K. S. Opiela, "The development of an enhanced finite element tire model for roadside safety hardware assessment," Proceedings of the Institution of Mechanical Engineers, Part K: Journal of Multi-Body Dynamics, vol. 226, no. 3, pp. 206-219, 2012.

[3] L. Chen, Y. Luo, M. Bian, Z. Qin, J. Luo, and K. Li, "Estimation of tire-road friction coefficient based on frequency domain data fusion," Mechanical Systems and Signal Processing, vol. 85, pp. 177-192, 2017.

[4] A. Pazooki, S. Rakheja, and D. Cao, "Modeling and validation of off-road vehicle ride dynamics," Mechanical Systems and Signal Processing, vol. 28, pp. 679-695, 2012.

[5] M. Matsubara, D. Tajiri, T. Ise, and S. Kawamura, "Vibrational response analysis of tires using a three-dimensional flexible ring-based model," Journal of Sound and Vibration, vol. 408, pp. 368-382, 2017.

[6] C.-L. Zhao and M.-Y. Zang, "Application of the FEM/DEM and alternately moving road method to the simulation of tiresand interactions," Journal of Terramechanics, vol. 72, pp. 27-38, 2017.

[7] I. J. M. Besselink, A. J. C. Schmeitz, and H. B. Pacejka, "An improved magic formula/swift tyre model that can handle inflation pressure changes," Vehicle System Dynamics, vol. 48, no. sup1, pp. 337-352, 2010.

[8] J. Li, Y. Zhang, and J. Yi, "A hybrid physical-dynamic tire/ road friction model," Journal of Dynamic Systems, Measurement, and Control, vol. 135, no. 1, 11 pages, 2013.

[9] C. Wei and O. A. Olatunbosun, "Transient dynamic behaviour of finite element tire traversing obstacles with different heights," Journal of Terramechanics, vol. 56, pp. 1-16, 2014.

[10] H. Yamashita, Y. Matsutani, and H. Sugiyama, "Longitudinal tire dynamics model for transient braking analysis: ANCFLuGre tire model," Journal of Computational and Nonlinear Dynamics, vol. 10, no. 3, 11 pages, 2015.

[11] D. Huang, L. Tang, and R. Cao, "Free vibration analysis of planar rotating rings by wave propagation," Journal of Sound and Vibration, vol. 332, no. 20, pp. 4979-4997, 2013.

[12] Z. Liu and Q. Gao, "Development and parameter identification of the flexible beam on elastic continuous tire model for a heavy-loaded radial tire," Journal of Vibration and Control, vol. 24, no. 22, pp. 5233-5248, 2018.

[13] T. D. Vu, D. Duhamel, Z. Abbadi, H.-P. Yin, and A. Gaudin, "A nonlinear circular ring model with rotating effects for tire vibrations," Journal of Sound and Vibration, vol. 388, pp. 245-271, 2017.

[14] C. Wang, B. Ayalew, T. Rhyne, S. Cron, and B. Dailliez, "Static analysis of a thick ring on a unilateral elastic foundation,"
International Journal of Mechanical Sciences, vol. 101-102, pp. 429-436, 2015.

[15] C. Wang, B. Ayalew, T. Rhyne, S. Cron, and B. Dailliez, "Forced in-plane vibration of a thick ring on a unilateral elastic foundation," Journal of Sound and Vibration, vol. 380, pp. 279-294, 2016.

[16] Z. Liu and Q. Gao, "Development of a flexible belt on an elastic multi-stiffness foundation tire model for a heavy load radial tire with a large section ratio," Mechanical Systems and Signal Processing, vol. 123, pp. 43-67, 2019.

[17] V. V. Krylov and O. Gilbert, "On the theory of standing waves in tyres at high vehicle speeds," Journal of Sound and Vibration, vol. 329, no. 21, pp. 4398-4408, 2010.

[18] R. J. Pinnington, "A wave model of a circular tyre. Part 1: belt modelling," Journal of Sound and Vibration, vol. 290, no. 1-2, pp. 101-132, 2006.

[19] Z. Liu, Q. Gao, and H. Niu, "Development of the flexible ring on an elastic continuous foundation tire model for planar vibration of the heavy load radial tire," Applied Sciences, vol. 8, no. 11, p. 2064, 2018.

[20] Y.-T. Wei, Z. Liu, F.-Q. Zhou, and C.-L. Zhao, "Threedimensional REF model of tire including the out-of-plane vibration," Journal of Vibration Engineering, vol. 29, pp. 795-803, 2016. 